\title{
The Improved Binary-Real Coded Shuffled Frog Leaping Algorithm for Solving Short-Term Hydropower Generation Scheduling Problem in Large Hydropower Station
}

\author{
Zhe Yang, Kan Yang $\mathbb{D}^{D}$, Lyuwen Su, and Hu Hu \\ College of Hydrology and Water Resources, Hohai University, Nanjing 210098, China \\ Correspondence should be addressed to Kan Yang; kyang@hhu.edu.cn
}

Received 7 March 2018; Revised 27 June 2018; Accepted 12 July 2018; Published 13 August 2018

Academic Editor: Konstantinos Karamanos

Copyright (C) 2018 Zhe Yang et al. This is an open access article distributed under the Creative Commons Attribution License, which permits unrestricted use, distribution, and reproduction in any medium, provided the original work is properly cited.

\begin{abstract}
The short-term hydro generation scheduling (STHGS) decomposed into unit commitment (UC) and economic load dispatch (ELD) subproblems is complicated problem with integer optimization, which has characteristics of high dimension, nonlinear and complex hydraulic and electrical constraints. In this study, the improved binary-real coded shuffled frog leaping algorithm (IBR-SFLA) is proposed to effectively solve UC and ELD subproblems, respectively. For IB-SFLA, the new grouping strategy is applied to overcome the grouping shortage of SFLA, and modified search strategies for each type of frog subpopulation based on normal cloud model (NCM) and chaotic theory are introduced to enhance search performance. The initialization strategy with chaos theory and adaptive frog activation mechanism are presented to strengthen performance of IR-SFLA on ELD subproblem. Furthermore, to solve ELD subproblem, the optimal economic operation table is formed using IR-SFLA and invoked from database. Moreover, reserve capacity supplement and repair, and minimum on and off time repairing strategies are applied to handle complex constraints in STHGS. Finally, the coupled external and internal model corresponding to UC and ELD subproblems is established and applied to solve STHGS problem in Three Gorges hydropower station. Simulation results obtained from IBR-SFLA are better than other compared algorithms with less water consumption. In conclusion, to solve STHGS optimization problem, the proposed IBR-SFLA presents outstanding performance on solution precision and convergence speed compared to traditional SFLA effectively and outperforms the rivals to get higher precision solution with improving the utilization rate of waterpower resources.
\end{abstract}

\section{Introduction}

Short-term hydro generation scheduling (STHGS), i.e., economic operation in hydropower station, including space and time optimization, is a typical dual optimization, multidimensional, noncontinuous, nonlinear problem with numerous complex hydraulic and electrical constraints. STHGS aims to find a profitable way to decrease water consumption and the cost of on and off transition between hydropower units with satisfying the power generation demand. The STHGS problem can be decomposed into two subproblems: the space optimization, namely, economic load dispatch (ELD), and the time optimization, the hydropower unit commitment (UC). The ELD subproblem attempts to reasonably determine the power output of each unit so as to minimize water consumption [1]. Efficiency, under different operation conditions, corresponding to each type of units is discrepant especially the large capacity unit. In addition, to keep unit operational within stable operation zone is beneficial to prolong the life of units. Therefore, to effectively distribute output for a startup unit to burden and focus energy on improving integrated units operation efficiency in stable operating zone are of vital importance in STHGS problem. The unit commitment (UC), aiming to pick up a wise startup and shutdown schedule of units during operational period, is 0-1 binary coded subproblem [2]. Along with the power generation, a large amount of water will be consumed in water turbine. Moreover, part of the water will be lost due to state change between startup and shutdown and frequent on and off transition is greatly harmful to service life of units [3]. Thus, a rational and highly efficient unit commitment can steeply minimize water consumption and simultaneously 
improve dependability of safe operation in hydropower system. In summary, to accurately describe and find reasonable unit commitment and economic load distribution can not only save the water resources and operation cost but also improve the turbine efficiency and service life. In order to efficiently utilize hydropower resources in reservoir and save limited water resources, it is meaningful and significant to build the model of STHGS and acquire feasible solution with effective method.

During the past few years, a number of traditionally mathematical methods have been put forward to handle STHGS problem. The traditional methods are focused on branch and bound algorithm [4] (Cohen and Yoshimura 1987), linear programming (LP) [5] (Jabr et al. 2000), mixed integer nonlinear programming [6] (Catalao J 2011), Lagrangian relaxation (LR) [7-10] (Oliveira et al. 1992; Ohishi T, W. Ongsakul; N. Petcharaks; Sum-Im et al. 2014; ChengC-P), quadratic programming (QP) [11] (Granelli and Montagna 2000), and dynamic programming (DP) [12-15] (Allen and Bridgeman 1986; Siu et al. 2001; Yi et al. 2003; Cheng et al. 2009), which have met with different degrees of success to STHGS. Nevertheless, methods mentioned have defects on mechanism, stability, and efficiency. Lagrangian multiplier is introduced into the objective function to structure dual problem to simplify calculation in the LR method; however, it is still difficult for coupling various constraints and finding a suitable multiplier. DP, though it can obtain the best solution theoretically, tends to face dimension disaster and costs plenty of computation time, which limits the application in dealing with STHGS issue. Therefore, the approximation of dynamic programming (ADP) algorithm is put forward to solve the problem of dimension disaster and overcomes these shortcomings to an extent [16] (Wei et al. 2014). On the whole, traditional methods encountered numerous obstacles when facing large scale power systems with huge installed capacity.

To overcome shortage of traditional methods, along with high-speed computer technology development, intelligent algorithms, i.e., heuristic stochastic methods, have been developed rapidly to exploit application in managing STHGS problem. These methods mainly include evolutionary programming (EP) [17] (Christober et al. 2011;), differential evolution (DE) [18, 19] (Lu et al. 2010; Mo et al. 2013), genetic algorithm (GA) [1, 20, 21] (Abido et al. 2003; Senthil Kumar V and Mohan M.R 2010; Zheng et al. 2013), tabu search (TS) [22] (Bakhta $\mathrm{N}$ et al. 2013), artificial neural network [23] (Sasaki H et al. 1992); particle swarm optimization (PSO) [24-26] (Yuan et al. 2009; Kumar et al. 2011; Zhao (2006)), chaotic optimization algorithm (COA) [27] (Cai et al. 2007), immune algorithm [28] (Wang and Yang 2010), ant colony optimization (ACO) $[29,30]$ (Vaisakh K and Srinivas L (2011); Chen et al. 2015), gravitational search algorithm (GSA) [31-34] ( Shen et al. (2015, 2016 a, b); Yuan X. et al. 2014; Li et al. 2015), and fireworks algorithm (FA) [35](K. Srikanth et al. 2016). Owing to flexible search strategies and powerful convergence capability, these algorithms can obtain higher quality solution with accepted optimization time to a certain extent in comparison with traditional methods. Furthermore, the mixed algorithm which combines superiority of several algorithms is introduced to accelerate computational speed and boost search ability for global optimum. However, intelligent heuristic stochastic methods also cause defects and imperfect constituent parts. The improved immune algorithm is applied to optimize the hydro generation scheduling, but the precision and efficiency need to further enhance. Hu et al. (2012) combined ant colony algorithm with equal incremental method; however, the operation efficiency drastically slows down with the increase in the number of units and parameter configuration may bring premature convergence trouble [36]. Wu (2015) adopts the measure of discrete particle swarm optimization (DPSO) with relevant improvement; however, global convergence is inefficient which makes it incapable of obtaining convincible solutions [37]. The gravitational search algorithm (GSA) proposed by Shen introduces the chaos theory to enhance local search ability, but the overall efficiency and robustness remain to be strengthened $[31,32]$. In summary, the intelligent heuristic stochastic algorithms mentioned present outstanding performance on computational accuracy, stability, and speed compared to that in traditional method. However, few methods may encounter premature convergence with limitations in local and global search strategies, making it difficult to find theoretical optimal solution. Therefore, it is significant and challengeable to develop effective method to realize more efficient and economical hydro generation scheduling.

The shuffled frog leaping algorithm (SFLA), a stochastic global optimization method, is inspired by predation behavior of frogs in wetland and first brought forward by Eusuff and Lansey in 2006 [38]. It is found that SFLA can guide the search toward global optimum direction by means of the global information exchange and internal communication mechanism [39]. SFLA, which is easy and convenient to code and has less control parameters, is verified to be compatible with handling comprehensive optimization problems, including the nonlinear and high dimensional discrete systems [40, 41] ( $\mathrm{Li}$ et al. 2014; Cao 2014). Li and Gao both adopt SFLA to solve the problem of economic dispatch and unit commitment and put forth targeted improvements to overcome drawback of SFLA, thus enhancing performance in searching and convergence ability. Nonetheless, the local update formula of SFLA still has limitations, leading the search degree of SFLA not enough to find the optimal solution and easy to be premature convergence, especially when all the frogs are concentrated in a local search field. In fact, the research concerning SFLA is still in the initial stage, in both theoretical and practical application especially the STHGS of hydropower station.

The paper focuses on effective improvements and strategies to overcome defects and strengthen performance of SFLA. However, the initial and fundamental SFLA is the real code version with continuous variable value, not appropriate for hydropower systems involving the discrete value ( 0 and 1 ) which represents online or offline status of hydropower units, respectively. For that reason, it is essential and necessary to transform real coded SFLA into the binary or discrete version BSFLA. Consequently, the transformational BSFLA is suitable for dealing with the $0 / 1$ programming problem, i.e., the discrete space of unit commitment and scheduling. 
Meanwhile, the real coded RSFLA is designed for the ELD problem. The improvements for BSFLA contained population initialization operated by renewed logistic chaotic map, renewed frog subpopulation division (leader, follower, and mutation frog), the cloud model for enhancing evolution and exploitation capability of leader and mutation frog subpopulations, and improved search strategies for follower frog subpopulations. Furthermore, the self-adaptive activation mechanism is introduced to the RSFLA. In summary, the improved discrete-real coded shuffled frog leaping algorithm (IBR-SFLA) is developed to solve the STHGS problem. The improved IB-SFLA is proposed as external search to optimize the UC subproblem; simultaneously the improved real coded SFLA (IR-SFLA) is as the internal search to handle ELD subproblem. Finally, the case study on Three Gorges hydropower station is used to test overall performance of IBR-SFLA. The simulation results show that it can figure out reliable high-quality solutions and outperform contrastive approaches and algorithms in precision and stability, while costing shorter time under the same operation condition.

\section{STHGS Problem Formulation}

As mentioned above, the model for STHGS includes two submodules: the UC and ELD. The aim of STGHS is to minimize, to the utmost, water consumption with meeting the power load demand, water balance, spinning reserve constraint, and other system constraints simultaneously in the duration of given operation periods, i.e., an hour for interval in a day.

2.1. Economic Load Dispatch (ELD). As one subproblem of STHGS, the ELD tends to pursue the minimum water consumption of generating power in hydropower station. The objective function can be described as

$$
\min Q=\sum_{i=1}^{n} u_{i} Q_{i}\left(H_{i}, P_{i}\right)
$$

$Q$ denotes the water consumption during operation periods; $u_{i}$ is the $i$ th hydro unit; $P_{i} \& H_{i}$ represent load for $i$ th unit to burden and water head for $i$ th unit, respectively; $Q_{i}\left(H_{i}, P_{i}\right)$ is the water consumption by $i$ th hydro unit.

Specifically, the water consumption function is set as quadratic function through curve fitting method. The function is expressed as

$$
Q_{i}\left(H_{i}, P_{i}\right)=a_{i}+b_{i} P_{i}+c_{i} P_{i}^{2}
$$

$a_{i}, b_{i}, c_{i}$ are coefficients in the fitting quadratic function and other parameters have the same meaning as the former.

2.2. Unit Commitment Problem. Reasonable hydropower units arrangement can bring enormous benefits on power generation efficiency. The basic goal of unit commitment is to effectively schedule the on/off state of hydropower units at the cost of minimum water which is consumed by power generation and switch process of startup and shutdown. The ascertained upstream and downstream water level, load demand, and various constraints are also taken into account [1, 30] (Zheng et al. 2013; Chen et al. 2015). Normally, the objective function can be signified as follows.

$$
\begin{gathered}
\min W=\sum_{t=1}^{T} \sum_{i=1}^{N}\left[Q_{i}^{t}\left(H_{i}^{t}, P_{i}^{t}\right) \cdot \Delta T \cdot u_{i}^{t}+u_{i}^{t} \cdot\left(1-u_{i}^{t-1}\right)\right. \\
\left.\cdot Q_{u p, i}+u_{i}^{t-1} \cdot\left(1-u_{i}^{t}\right) \cdot Q_{d n, i}\right]
\end{gathered}
$$

$W$ denotes total water consumption of hydropower units composed of power generation and startup and shutdown costs of units; $Q_{\mathrm{up}, i}, Q_{\mathrm{dn}, i}$ are startup and shutdown water consumption, respectively. $\Delta T$ is interval, i.e., one hour; $T$ is the total scheduling periods; $H_{i}{ }^{t}$ indicates water head for $i$ th unit at $t$ th interval; $P_{i}^{t}$ denotes power output for $i$ th unit at current $t$ th interval; $Q_{i}^{t}\left(H^{t}, P_{i}^{t}\right)$ is the outflow of the $i$ th unit at $t$ th interval corresponding to water head $H^{t}$ and power output $P_{i}^{t} ; u_{i}^{t}$ valuing 1 and 0 , respectively, indicates startup and shutdown state of $i$ th unit.

2.3. Constraints. As is mentioned above, there are a number of various hydraulic and electrical constraints in STHGS problem. It can be summarized as follows:

\section{(1) Power Balance Constraint}

$$
\sum_{i=1}^{\mathrm{N}} u_{i}^{t} \cdot P_{i}^{t}=P_{D}^{t}
$$

$P_{D}^{t}$ is the power demand at $t$ th interval of hydropower station; $u_{i}^{t}$ and $P_{i}^{t}$ have the same meaning as the former.

(2) Spinning Reserve Constraint

$$
\sum_{i=1}^{\mathrm{N}} u_{i}^{t} \cdot P_{i, \max } \geq P_{D}^{t}+P_{R}^{t}
$$

$P_{R}^{t}$ denotes spinning reserve at $t$ th interval.

(3) Hydropower Unit Output Limits

$$
P_{i, \min } \leq u_{i}^{t} \cdot P_{i}^{t} \leq P_{i, \max }
$$

$P_{i, \min }$ and $P_{i, \max }$ signify minimum and maximum load output of $i$ th unit, respectively.

\section{(4) Prohibited Operating Region Constraint}

$$
\begin{gathered}
P_{i, \min } \leq P_{i}^{t} \leq\left(P o z_{i}^{1}\right)^{l} \\
\left(\operatorname{Poz}_{i}^{m-1}\right)^{u} \leq P_{i}^{t} \leq\left(P o z_{i}^{m}\right)^{l} \\
\left(P o z_{i}^{r}\right)^{u} \leq P_{i}^{t} \leq P_{i, \max } \\
m=2,3 \ldots, r
\end{gathered}
$$

$r$ represents total prohibited operation zones, with $m$ to number them. $\left(P o z_{i}^{m}\right)^{l}$ and $\left(P o z_{i}^{m}\right)^{u}$ are lower and upper 
limits corresponding to $m$ th prohibited operation zone for the $i$ th unit.

(5) Minimum Up and Down Time Constraint

$$
\begin{gathered}
T_{\mathrm{i}, \text { on }}^{t} \geq T_{i, \text { up }} \\
T_{\mathrm{i}, \text { off }}^{t} \geq T_{i, \text { down }}
\end{gathered}
$$

$T_{i, u p}$ and $T_{i, \text { down }}$ are the minimum online (up time) and offline (down time) limits, ensuring stable and safe operation of hydropower units; $T_{\mathrm{i}, \text { on }}^{t}$ and $T_{\mathrm{i}, \text { off }}^{t}$ denote accumulated up/down time for $i$ th unit that had been online/offline till $t$ th interval.

(6) The Reservoir Storage and Flow Discharge Limits

$$
\begin{aligned}
& V_{t}^{\min } \leq V_{t} \leq V_{t}^{\max } \\
& Q_{t}^{\min } \leq Q_{t} \leq Q_{t}^{\max }
\end{aligned}
$$

$V_{t}^{\min }$ and $V_{t}^{\max }$ signify the minimum and maximum storage volume of hydropower station. Correspondingly, $Q_{t}^{\min }$ and $Q_{t}^{\max }$ are lower and upper limits of water discharge, respectively.

\section{(7) The Water Balance Constraint}

$$
V_{t}=V_{t-1}+\left(I_{t}-Q_{t}\right) \cdot \Delta t
$$

$V_{t}$ is the storage volume of reservoir at $t$ th interval; $I_{t}, Q_{t}$ are inflow and outflow (water release); $\Delta t$ is interval.

\subsection{Influencing Factors in STHGS}

2.4.1. The Stable Operation Region. As is demonstrated in Figure 1, the operation area of hydropower unit can be classified into three components: prohibition operation, limited operation, and stable operation regions or zones. Constraint (4) mentioned describes the prohibited operation limits of turbine-unit. Enormous vibration and noise will emerge when hydro unit operates in the prohibited operation zone (POZ) which brings great harm to unit security and stability. Therefore, to ensure long-term security and prolong the service life of units, it is vital to regulate unit operation zone and limit its operation within stable operation zone (SOZ). The dark blue area in Figure 2 shows the unit SOZ of Three Gorges left bank (VGS and ALOSTOM) under different water heads.

2.4.2. The Unit Operation Efficiency. The unit operation efficiency (UOE), another important turbine-unit characteristic, has a significant impact on generation and total water consumption. Specifically, the relationship between power generation and UOE can be expressed as the following formula:

$$
P=9.81 \eta Q H
$$

$\eta$ denotes unit operation efficiency (\%). $Q$ is water discharge and $H$ is effective water head. $P$ represents the power output $(\mathrm{kW})$.

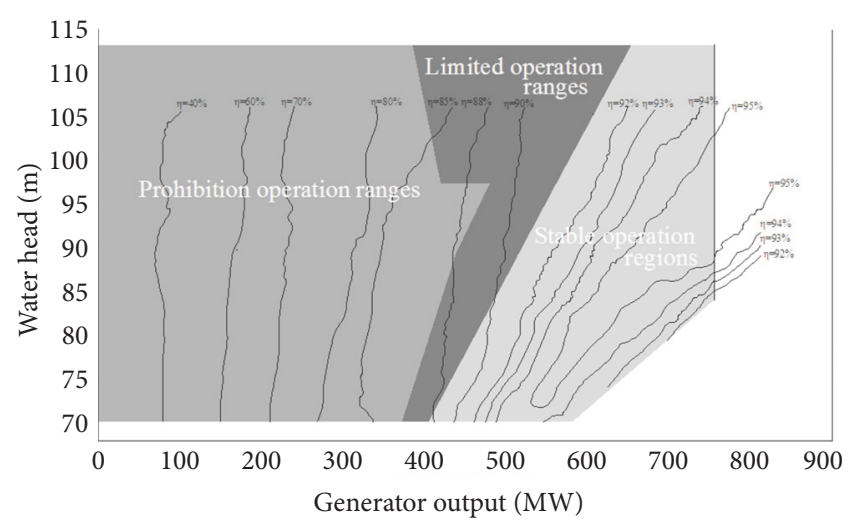

FIgURE 1: The operation region of left-bank ALSTOM units in Three Gorges hydropower station.

Under a certain water head, different unit power output corresponds to various efficiency which is shown in Figure 3. The curves in Figure 3, displaying UOE in Xiluodu hydropower station, do not present tendency of monotonic increasing but a sharp reduction after the maximum point [42]. The variation trend of curves is consistent with Figure 1. By analyzing the UOE curves under different operation zones in Figure 1, we can safely conclude that a single unit will have a relative higher efficiency when working under the stable operation zone (SOZ) than that in other zones. In this paper, the UOE and SOZ are fully considered into the ELD subproblem to fully fulfill purpose of minimizing water consumption.

2.4.3. The Unit Water Consumption Rate. The definition of hydropower unit water consumption rate (UWCR) is the water consumption corresponding to unit power output. Generally, it can be acquired by the following formula:

$$
C=\frac{Q_{i}\left(P_{i}, H_{i}\right)}{P_{i}}=\frac{1}{\eta H_{i}}
$$

where $Q_{i}\left(P_{i}, H_{i}\right)$ denotes generation flow. $C$ is water consumption rate of $i$ th unit when power output is $P_{i}$ and net water head is $H_{i}$.

From formula (12), we can see the UWCR is concerned with net water head $H_{i}$ and $\eta$ (UOE), of which differences are great among different units. The UWCR is introduced as an influencing factor, aiming to preferentially start up unit with lower UWCR and shut down unit with higher UWCR. Thus, the water consumption can be saved and spinning reserve constraint (constraint (2)) is satisfied simultaneously.

\section{The Introduction of IBR-SFLA and Model Establishment for STHGS Problem}

3.1. The Overview of Traditional SFLA. Inspired by foraging behavior about frogs in the swamp, the SFLA is a metaheuristic optimization based on swarm intelligence, combining deterministic method with random measure. It 

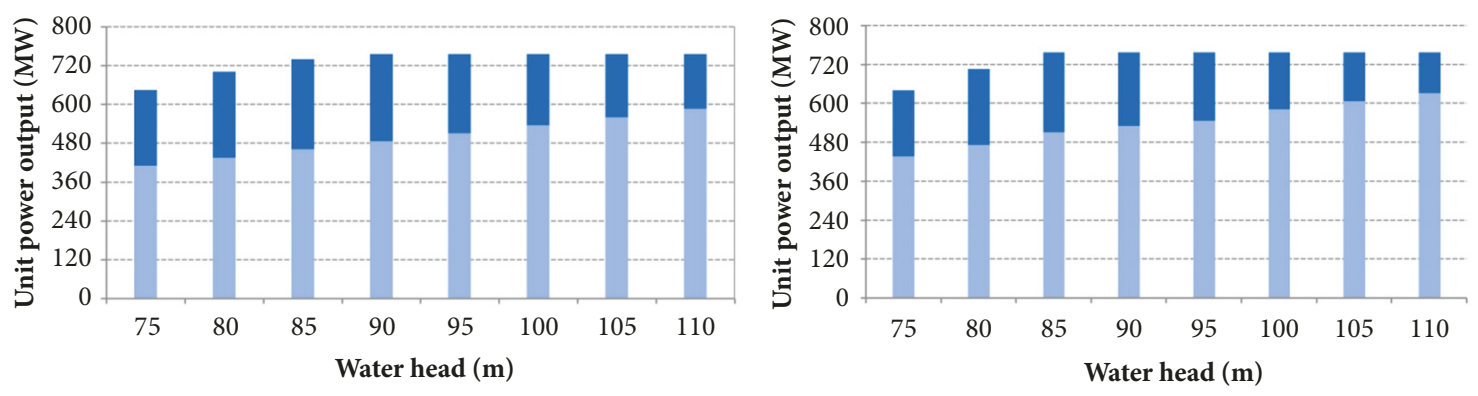

VGS units

ALSTOM units

FIgURE 2: The SOZ of left-bank units in Three Gorges hydropower station.

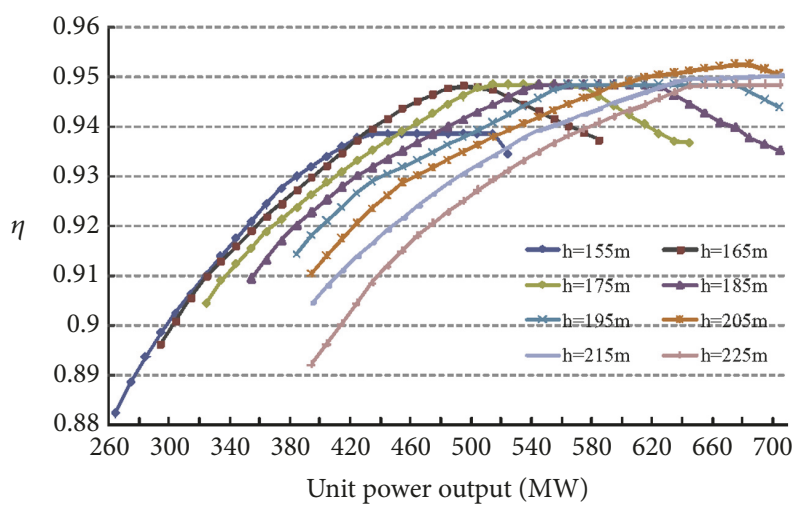

FIGURE 3: The unit operation efficiency (UOE) of right-bank unit in Xiluodu hydropower station.

incorporates advantage of memetics evolution and particle swarm algorithm which possesses characteristic of less parameters and easiness to comprehension [43] (Zhao and Chen 2016). The frog population is partitioned into a number of parallel subpopulations (communities), of which frogs are arranged to search prey and evolve independently, i.e., memetic evolution. Frogs in each subpopulation can be seen as potential feasible solutions and idea toward a goal and are infected by ideas of other frogs [44] (Zou et al. 2012). Memetic evolution aims to improve quality of individual frog and enhance performance, thus, directing frogs to exploit more excellent ideas and solutions. After a number of memetic evolution steps, the shuffling process, i.e., mingling frogs from different subpopulations, is activated to boost frog quality after exchanging information with each subpopulation so as to pass down the superior ideas [45](Luo et al. 2015).

Generally, the frog population division strategy can be described as follows: to begin with, the initial population will be ranked in descending order based on fitness of each frog. Then, the frogs are grouped into $m$ subpopulations which satisfy $M=m \times h$; i.e., each subpopulation contains $h$ frogs. Finally, the first frog will be assessed in first subpopulation and the next one is chosen to the next subpopulation, and so forth, until the $m$ th frog is grouped in the $m$ th subpopulation. The subpopulations are allocated with frogs in the first round, then the $(m+1)$ th frog is divided into the first subpopulation, the $(m+2)$ th frog is divided into the second subpopulation, and so on [39] (Sun et al. 2016). The rest of subpopulations adopt similar grouping measure to make all frogs classified so as to find the optimal and worst frog $u_{b}$ and $u_{w}$ within each subpopulation as well as the current global optimal frogs $u_{g}$ in the whole population.

The local search in each subpopulation merely renovates the worst frog individual in frog subpopulations. Usually, the position of frog is updated by the following formula:

$$
\begin{aligned}
d_{i} & =\operatorname{rand}() *\left(u_{b}-u_{w}\right) \\
u_{\text {wnew }} & =u_{w}+d_{i} \quad d_{\min } \leq d_{i} \leq d_{\max }
\end{aligned}
$$

rand ( ) denotes random function generating the number in the interval $[0,1] ; d_{i}$ is leap step of frog, $i=1,2,3 \ldots \mathrm{m}$; $d_{\min }$ and $d_{\max }$ are minimum and maximum frog leap step, respectively; $u_{b}$ and $u_{w}$ denote the best and worst frog positions of each subpopulation, respectively; $u_{\text {wnew }}$ is the new updated worst frog position.

The position of $u_{w}$ will be replaced by the new frog position of $u_{\text {wnew }}$ if the fitness of renewed frog $u_{\text {wnew }}$ is superior to that of previous one $u_{w}$. Otherwise, new frog position is acquired once through (13), in which $u_{b}$ is replaced by $u_{g}$. Then, if the process is still incapable of improving performance of worst frog $u_{w}$, a new frog $u_{\text {wnew }}$ is obtained randomly to displace the worst frog position $u_{w}$. All frogs are mixed together and rearranged in the frog shuffling process when local search is accomplished in all subpopulations. Then, the local evolution and global shuffling will perform repeatedly until the maximum iterations preset or convergence criteria are satisfied [44].

3.2. The Improved Binary Coded SFLA (IB-SFLA). The UC is a discrete subproblem which involves $0-1$ state variables. Therefore, the binary coded SFLA with improvements is adopted to find commitment schedule (unit on/off state).

3.2.1. The Grouping Strategy. Usually, the frogs are sorted in a descending order according to fitness in the traditional SFLA. Thereafter, the frogs are divided into subpopulations in order. Consequently, relatively worst frogs are sorted into last subpopulation. The population grouping limitation makes the worst frog in last subpopulation have difficulty in acquiring the excellent information and improving its quality. 
To avoid this limitation, the frog grouping process is updated. The frog population is divided into three types of memetic subpopulations: leader frog, follower frog, and mutation frog. As the elite individual in whole population, the leader frog subpopulation plays a role in exploring more superior position. Follower frog tends to share information and mutation frog exploits solution scope of unknown area. Frogs in each type of subpopulation experience evolution with different strategies or methods. Therefore, the relative worst frog can better develop new idea with evolution strategy suitable for itself. Meanwhile, the elite information exchange and communication between parallel memetic subpopulations will be more effective. The grouping method is elaborated as follows:

Step 1. Select the optimal frogs in all subpopulations and calculate average fitness of these frogs $F\left(u_{b}^{a v g}\right)$.

Step 2. Obtain the optimal frogs whose fitness is superior and inferior to $F\left(u_{b}^{a v g}\right)$ in all subpopulations. Then figure up average fitness of available frogs $F\left(u_{b}^{\text {bavg }}\right)$ and $F\left(u_{b}^{\text {wavg }}\right)$, respectively.

Step 3. Count number of subpopulations in which fitness of the optimal frog is superior to $F\left(u_{b}^{b a v g}\right)$ and inferior to $F\left(u_{b}^{\text {wavg }}\right)$, respectively. The number is stored in variables $m_{l e d}$ and $m_{\text {mua }}$; specifically, $m_{l e d}$ is the number of leader frog subpopulations and $m_{m u a}$ denotes number of mutation frog subpopulations.

Step 4. Set up total number of subpopulations $m$. The number of leader and mutation frog subpopulations has been acquired in Step 3. Therefore, the number of follower frog subpopulations is $m-m_{\text {led }}-m_{m u a}$.

3.2.2. The Modified Search Strategy for Followers Frog Subpopulation. In the improved binary coded SFLA (IB-SFLA), the frog position $u$ is discretized and coded with values 1 or 0 (on/off), while the frog leap step $d$ need not be discretized. The values of $d$ determine whether $u$ is 1 or 0 in the form of probability. In this paper, the improved sigmoid function is developed to build relationship between frog position $u$ and leap step $d$. The function details are defined in (15) and graph of function is shown in Figure 4.

$$
\begin{aligned}
& u_{w, t+1}^{\text {new }}[i, j, k] \\
& = \begin{cases}1 & \text { if } d_{t}[i, j, k] \geq 0 \text { and } r \leq \frac{2}{1+e^{-d_{t}[i, j, k]}}-1 \\
u_{w, t}^{\text {old }}[i, j, k] & \text { ortherwise } \\
0 & \text { if } d_{t}[i, j, k] \leq 0 \text { and } r \leq 1-\frac{2}{1+e^{-d_{t}[i, j, k]}}\end{cases}
\end{aligned}
$$

$d_{t}[i, j, k]$ is the leap step of $k$ th unit attached to the worst frog in $i$ th subpopulation at $j$ th period; $u_{w, t}^{\text {old }}[i, j, k]$ is the old location of the worst frog in previous $t$ th iteration; $u_{w, t+1}^{n e w}[i, j, k]$ is location of the renewed worst frog in $i$ th subpopulation in the $t+1$ th iteration.

Furthermore, in the frog evolution process of traditional SFLA mentioned above, worst frog in subpopulation is

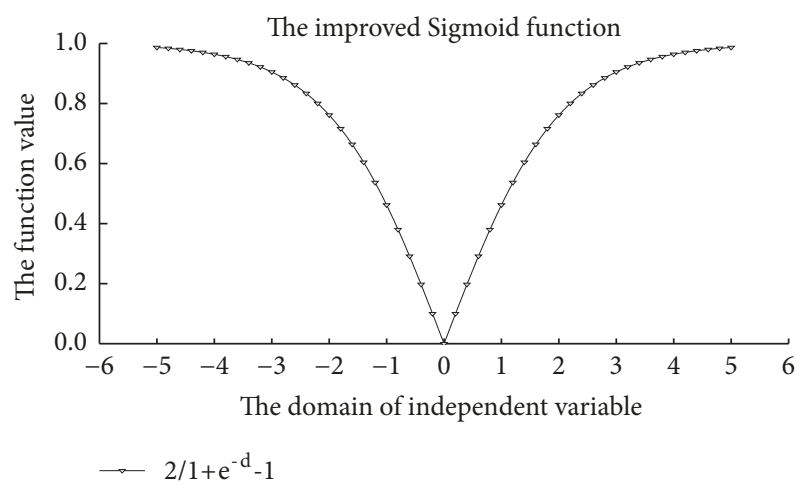

FIGURE 4: The graph of improved sigmoid function.

updated primarily according to the best frog $u_{b}$ in subpopulation. If the new position of the worst frog is still worse than before, frogs are evolved according to the global optimal frog $u_{g}$ in (13). Under this evolution strategy, the information is so scarce for worst frog that it is difficult to develop new idea to the fullest extent. Therefore, the old position updating formula is modified to fully pass down the information and novel idea of $u_{b}$ and $u_{g}$ simultaneously. The new updating strategy is shown in (16) and conducted if the new frog is not to be improved after traditional evolution process. The frog position evolution is still according to (15).

$$
d_{t+1}=\beta_{1} *\left(u_{b}-u_{w}\right)+\beta_{2} *\left(u_{g}-u_{w}\right)
$$

$d_{t+1}$ is the renewed frog leap step; $u_{b}$ and $u_{w}$ are location of the best and worst frogs, respectively; $u_{g}$ denotes the best location in whole frog population currently. Both $\beta_{1}$ and $\beta_{2}$ are $\operatorname{rand}()$ function which creates random number in the interval of $[0,1]$.

\subsubsection{The Elite Evolution Strategy for Leader Frog Subpopula-} tion Based on NCM. The cloud theory was put forward by $\mathrm{Li}$ et al. in 1995, which possesses the prominent characteristics of randomness and stable tendency, and it has been proved to avoid the local extreme to a certain degree [46]. In recent years, the cloud model has been introduced and combined with a number of algorithms such as GA, PSO, and evolution algorithm [47-49] (Dai et al. 2007; Zhang et al. 2008; Zhang et al. 2012). The introduction of cloud model algorithm can effectively improve overall search algorithm performance and contribute to overcome the defects on easily falling into premature trap to some extent.

According to the principle of sociology, the more excellent individual often appears around the current optimal individual in subpopulations. In other words, the better frog is found with great probability in the vicinity of the current best frog. Therefore, the normal cloud model is introduced into SFLA to seek the potential best frog within a feasible zone and reduce possibility of trapping in the local optimum.

Normal cloud model (NCM) is brought forth to describe the uncertain conversion relation of qualitative concept or qualitative knowledge with its quantitative expressions. The cloud model is proposed for handling combination of 
qualitative and quantitative problem. The model reflects the correlation of randomness and fuzziness, establishing the mapping relation with quantitativeness and qualitativeness. $U$ is defined as the domain which is one-dimensional or multidimensional. $C$ is a qualitative concept corresponding to $U$. For a certain element $x \in U$, a random number with stable tendency $F=C(x)$ is defined as certainty of $x$ to $C$. The distribution of $x$ in the domain $U$ is called cloud model and each $x$ is one cloud droplet in the domain space. The expectation $E_{x}$, entropy $E_{n}$, and hyperentropy $H_{e}$ are used for characterizing the digital features of cloud [50] (Ma et al. 2013). The details on $E_{x}, E_{n}$, and $H_{e}$ are as follows:

(1) $E_{x}$ is central of all droplets and the most representative cloud droplet for qualitative concept. The optimal frog in leader frog subpopulations is selected as the representative cloud droplet.

(2) The entropy $E_{n}$ denotes the uncertainty measurement of qualitative concept. It is not merely reflecting emergence randomness of cloud droplet but also revealing the correlation of fuzziness and randomness. The scope of generating cloud droplet and its randomness highly depends on the value of $E_{n}$; specifically the larger $E_{n}$ is, the more obvious the randomness is and the more broader the scope is. $E_{n}$ is valued with the standard deviation of the frog fitness in subpopulation during current iteration.

(3) The hyperentropy $H_{e}$ determined by randomness and fuzziness represents measurement of entropy, i.e., the entropy of entropy. To boost search randomness in initial iteration stage and maintain search stability in the later stage, $H_{e}$ is relevant to $E_{n}$ with value of $E_{n} / 5$.

The process of creating cloud droplet according to basic normal cloud generator is detailed as follows [39, 51] (Ding and Wang 2013; Sun et al. 2016).

Step 1. Generate a normal random number $E_{n n}$ with expectation $E_{n}$ and standard deviation $H_{e}$.

Step 2. Thereafter, generate a normal random number $x$ as a cloud droplet, which is taken $E_{x},\left|E_{n n}\right|$ as expectation and standard deviation, respectively.

Step 3. The calculation $E_{n n}$ and $x$ are plugged into formula $F=e^{-\left(x-E_{x}\right)^{2} / 2\left(E_{n n}\right)^{2}}$ to figure up certainty pertaining to the qualitative concept $C$.

Step 4. Repeat the above-mentioned processes until the cloud droplets satisfy the terminal condition.

The optimal individual in leader frog subpopulation is regarded as a normal droplet Cloud $\left(E_{x}, E_{n}, H_{e}\right)$. Then, several cloud droplets are created according to this droplet by normal cloud generator. The number of new created cloud droplets is the same with capacity of subpopulation $h$. The new droplet replaces original optimal frog if fitness is better than before. Otherwise, the original optimal frog is still to be retained [52] (Zhang and Li 2015). The elite evolution strategy aims to evolve the best individual in subpopulation, while the worst frog adopts the traditional updating mechanism to finish evolution.
3.2.4. The Mutation Strategy for Mutation Frog Subpopulation. There are variation phenomena in the biosphere, which has characteristic of randomness and burstiness. However, the biomutation is beneficial to track the potential optimal individual in the unknown solution space and avoid falling into local optimum to some extent.

Through analysis of the grouping strategy, it is known that relative worst frogs are sorted into the mutation subpopulations. In comparison with other frog subpopulations, the mutation frog is weaker than others to make contribution to developing novel idea. Therefore, the mutation frogs are designed to find profitable variation and corresponding update strategy for mutation frogs is adaptively changed using chaotic mapping method.

The logistic map is a typical discrete-time dynamical systems, i.e., nonlinear equation. The chaotic sequence is produced by logistic map, which is seemingly chaotic but with regularity and ergodic property. In this paper, the modification is developed for conventional logistic map to strengthen population diversity and distribution uniformity. The chaotic sequence is calculated by the following reformative logistic map equation [53] (Zou et al. 2016):

$$
x_{j}^{t+1}=1-2\left(x_{j}^{t}\right)^{2} \quad x_{j}^{t} \in[-1,1] ; \mathrm{t}=1,2 \ldots \mathrm{t}_{\max }
$$

$j$ represents decision variable dimension; $t$ is the current iteration; $t_{\max }$ is maximum iterations; variable $x_{j}^{t}$ is defined as the $j$ th chaos variable after $t$ th iteration, valuing in the interval $[-1,1]$.

Each dimension of chaotic variables $x_{j}$ is reflected to feasible solution space of optimization variable, i.e., frog step $d_{i j}$ by (18). Then, the conversion relation between mutation frog leap step and position $u$ is established through (15).

$$
d_{i j}=\frac{\left(x_{j}^{t}+1\right)}{2} *\left(d_{\max }-\left(-d_{\min }\right)\right)+\left(-d_{\min }\right)
$$

If the new position $u_{\text {new }}$ of mutation frog obtains a better performance than the overall optimal frog, the position of current optimal frog is replaced by this position. Otherwise, calculations in (17) and (18) are repeated until the given terminal condition is satisfied. The new individual created by mutation strategy based on chaotic mapping possesses characteristic of randomness, fuzziness, and determinacy. This mutation strategy contributes to effectively exploit space beyond local convergence domain, thus guiding algorithm to locate the more outstanding frog.

3.3. The Improved Real Coded SFLA (IR-SFLA). The ELD subproblem is a continuous, highly nonlinear, and multidimensional optimization problem, aiming at efficiently dispatching power load of hydropower units. An optimal dispatch schedule is beneficial to minimize water consumption. Because the power load is continuous, the real coded mechanism of SFLA is suitable for handling ELD subproblem. However, the evolution strategy in traditional SFLA is liable to slow down convergence speed and not easily escape from local optimum. Thus, several changes for the original real coded version of SFLA (IR-SFLA) are introduced in this paper. 
3.3.1. Initial Population Based on Chaos Theory. The initialization has a significant effect on convergence speed and final solution quality. Traditional population initialization is accomplished through the random guided search strategy, making it difficult to locate feasible zone. Chaos theory possesses inherent stochastic characteristic and ergodicity, which is highly sensitive to initial value and liable to escape from local optimum. Therefore, the improved chaos logistic map is applied to frog population initialization to strengthen population diversity and distribution uniformity during the initialization process. It is beneficial to promote overall quality of initial population and locate feasible search zone effectively [53, 54] (Cheng et al. 2008; Zou et al. 2016).

Similarly, the chaotic sequence $x_{j}$ creation process is according to (17), and chaotic variables are mapped to feasible solution space of variable $y_{i j}$ on the basis of (18). In IR-SFLA, variable $y_{i j}$ is real coded, which denotes the power load in ELD subproblem and the frog position updating formula is in line with (14). Then, calculate fitness $f\left(y_{i j}\right)$ of $y_{i j}$ which is concerned with water consumption. If $f\left(y_{i j}\right)^{k} \leq f\left(y_{i j}\right)^{k+1}$, i.e., the new position is superior to the old created in the last generation, then $f\left(y_{i j}\right)^{k}=f\left(y_{i j}\right)^{k+1}$ and $y_{i j}{ }^{k}=y_{i j}{ }^{k+1}$. The process is repeated in a number of iterations and select the optimal frogs into initial population.

3.3.2. The Improved Search and Position Evolution Strategy. As mentioned in IB-SFLA, the information is too scarce to guide the update of worst frog with using search strategy of traditional SFLA. Similarly, the information of local optimal frog $u_{b}$ and overall optimal frog $u_{g}$ is combined to lead the worst frog into novel position. Furthermore, drawing on the $\mathrm{PSO}$, the history frog leap step is taken into consideration to further enrich update information of worst frog. The improved search strategy is shown in the following equation, and the position updating is according to (14).

$$
d_{t+1}=\theta d_{t}+\beta_{1} *\left(u_{b}-u_{w}\right)+\beta_{2} *\left(u_{g}-u_{w}\right)
$$

$u_{b}, u_{w}, u_{g}$ are real coded with continuous variable, i.e., unit power load in ELD. Similarly, $d_{t}$ denotes historical frog leap step; $\beta_{1}$ and $\beta_{2}$ are evenly distributed random number between $(0,1) ; \theta$ is adjustment coefficient, which aims at controlling leap step and convergence speed in order to achieve balance between local deep-searching and global information shuffling process. In this paper, the improved adjustment coefficient equation is shown in

$$
\begin{aligned}
\theta= & \left(\theta_{\max }-\theta_{\min }\right) \\
& \cdot\left[\gamma * \frac{\left(L_{g}-L_{t}\right)}{L_{g}}+(1-\gamma) * \frac{\left(1-t / L_{s}\right)}{\left(1+t / L_{s}\right)}\right] \\
& +\theta_{\min }
\end{aligned}
$$

$L_{t}$ is the number of global shuffling iterations; $L_{g}$ is total global shuffling iterations; $t$ is the number of local iterations in memetic subpopulations; $L_{s}$ represents total local iterations in subpopulations; $\theta_{\max }, \theta_{\min }$ are the maximum and minimum adjustment coefficient; $\gamma$ is weight coefficient in the interval of $(0,1)$.

At the initial evolution stage, the adjustment coefficient $\theta$ is assigned with a larger value to boost search capacity in global solution space and a lower value at the later evolution stage. A lower adjustment coefficient $\theta$ slows down the evolution speed and promotes depth search in local feasible solution space in the later stage [55].

3.3.3. The Adaptive Frog Activation Mechanism. The local optimal individual $u_{b}$, overall optimal $u_{g}$, and worst frog $u_{w}$ to be updated are very close with great possibility if frog population search and evolve in a narrow space, i.e., local optimum. Assuming that the difference of $\left(u_{g}-u_{b}\right)$ is close to zero, population diversity is reduced and new frog position update for $u_{w}$ will weaken and even stagnate. Therefore, the adaptive frog activation mechanism is introduced into frog update and position evolution process. The activation mechanism replaces inactive frog lack of evolution ability with creating a new individual. Consequently, search ability of $u_{w}$ is reactivated and new position tends to be found. The new frog creation method is expounded as follows.

$$
\begin{aligned}
\text { new } u_{w}= & u_{w}(1+\theta \cdot \beta), \quad \text { if }\left|F\left(u_{g}\right)-F\left(u_{b}\right)\right| \leq \varepsilon \\
\theta= & \theta_{e}+\arctan \left(1.56\left(1-\left(\frac{t * L_{t}}{L_{s} * L_{g}}\right)^{\varphi}\right)\right) \\
& *\left(\theta_{s}-\theta_{e}\right)
\end{aligned}
$$

$\beta$ is random number in the range of $[0,1] ; \theta$ denotes adjustment coefficient which uses the arc tangent function to make it changeable dynamically; $\theta_{s}$ and $\theta_{e}$ are initial and final value of $\theta$ which are evaluated with 0.9 and 0.4 , respectively; $\varphi$ represents controlling factor in the range of $[0.4,0.7] ; t, L_{t}$, $L_{s}$, and $L_{g}$ are defined in (20). To maintain fast convergence characteristic of IR-SFLA and reduce uncertainty of random number, a relative lower $\theta$ is used in the later iteration stage. $\left|F\left(u_{g}\right)-F\left(u_{b}\right)\right| \leq \varepsilon$ is decision condition; $\varepsilon$ is a positive number defined as similarity threshold; $F\left(u_{g}\right)$ and $F\left(u_{b}\right)$ are fitness of $u_{g}$ and $u_{b}$, respectively. Equation (21) is activated if difference of $F\left(u_{g}\right)$ and $F\left(u_{b}\right)$ satisfies the decision condition in a certain number of iterations.

3.3.4. The Numerical Simulation Experiment. Numerical simulation experiment is designed to demonstrate the superiority of IR-SFLA in solving continuous space optimization problem. The typical Sphere, Rosenbrock, Rastrigin, Griewank, and Schaffer benchmark problems with 30 dimensions are selected as simulation experiment. The IR-SFLA is compared with PPNGA, EACO, SFLA, CGSA, and IBCO, and 20 independent simulations are conducted for all algorithms. Partial results of the 20 simulations are shown in Figure 5. The Mean and Standard Deviation (STD) acquired from 20 independent simulations are listed in Table 1, respectively. In addition, the Success Rate and Mean Number of Iteration Generation (MNIG) required to achieve convergence precision preset are introduced to compare convergence 

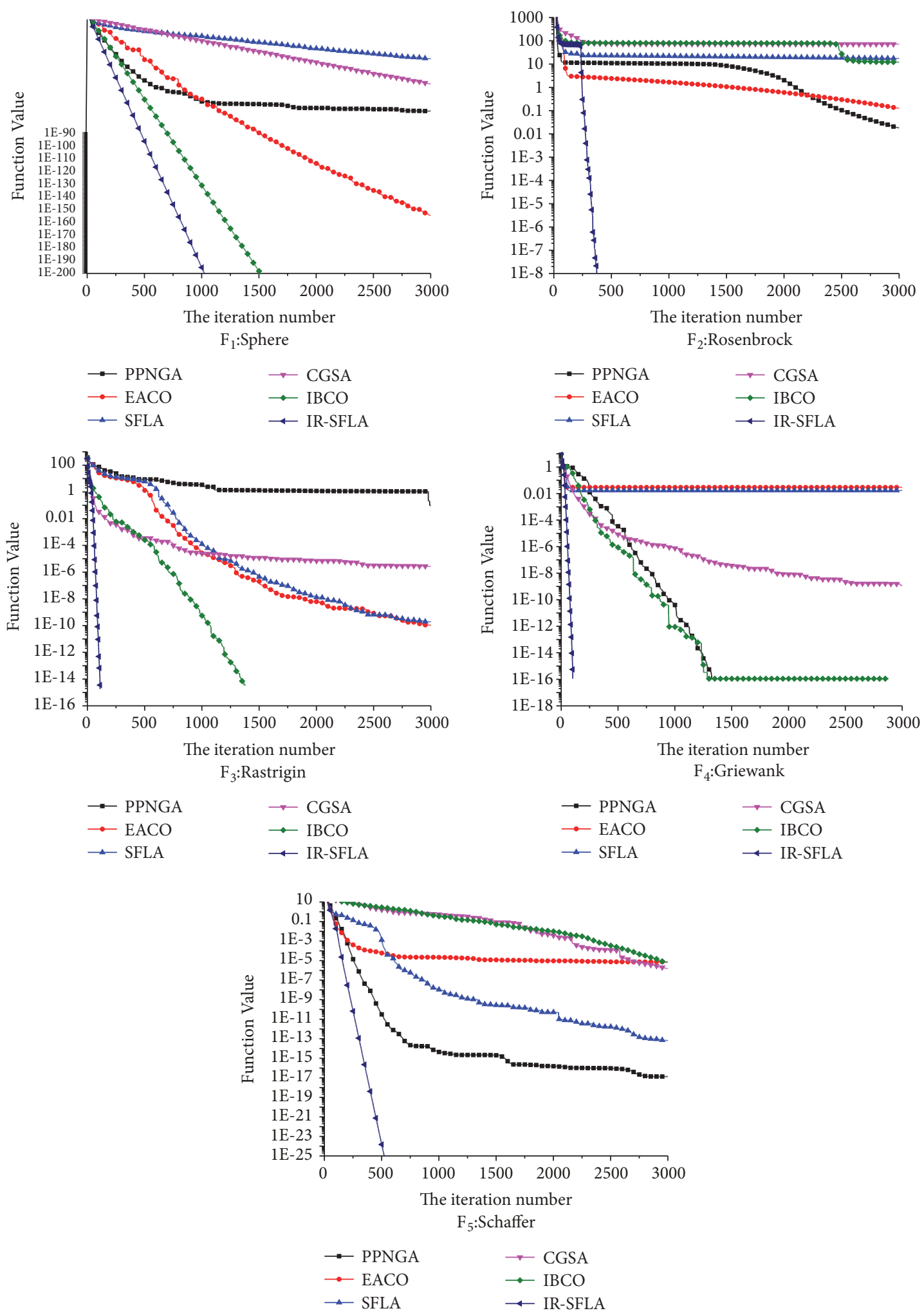

FIGURE 5: The iterative curve of IR-SFLA and compared algorithms for test benchmark functions. Notes: the algorithms convergent to the optimal result " 0 " after stagnation point. 


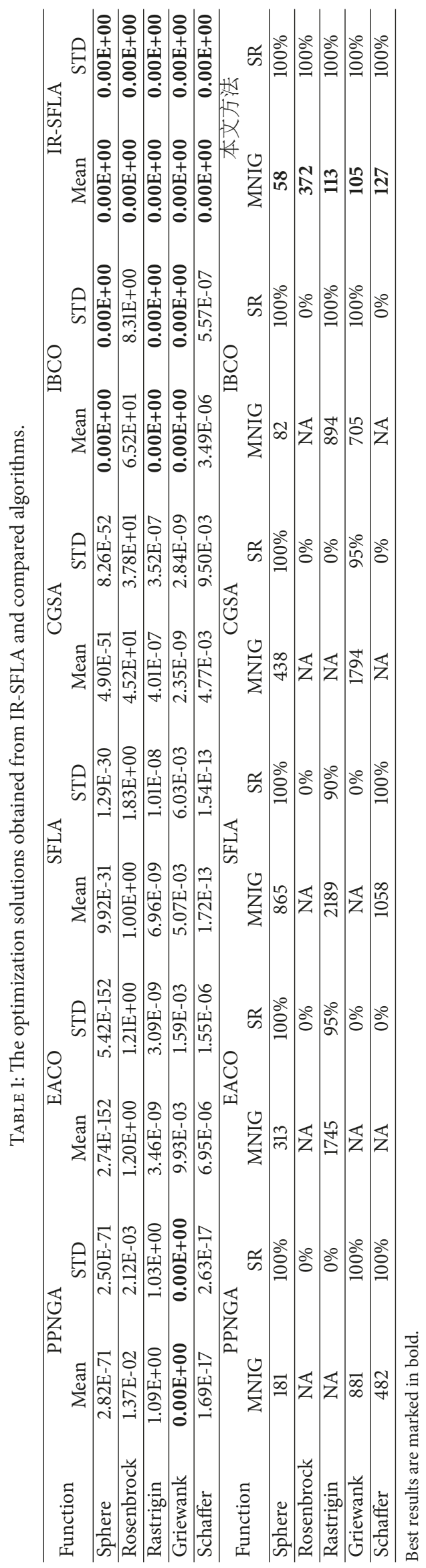


speed and reliability of each algorithm, respectively. The results are also listed in Table 1 where the "NA" denotes "Not Applicable" and the convergence is set as $10^{-8}$ for all the benchmarks. To further compare IR-SFLA with other rivals, the Friedman and Kruskal-Wallis tests are used to depict the algorithm superiority and listed in Table 2.

Through inspection of the iteration curves in Figure 5 and results of Mean and STD in Table 1, it is clearly revealed that IR-SFLA eventually converges to the evidently beat solution for each benchmark problem in comparison with other algorithms. Furthermore, the performance on convergence speed for IR-SFLA is much better than competitors especially in terms of $\mathrm{F}_{2}$ Rosenbrock, $\mathrm{F}_{3}$ Rastrigin, and $\mathrm{F}_{4}$ Griewank. For $F_{1}$ Sphere, $F_{3}$ Rastrigin, and $F_{4}$ Griewank, the performance for IBCO is outstanding and converges to the best solution; however, the convergence speed for IBCO has no advantage compared with IR-SFLA through the iteration curves in Figure 5. Moreover, the IBCO is not skilled in solving $\mathrm{F}_{2}$ and $\mathrm{F}_{5}$ benchmarks from the aspects of final precision and stability reflected by STD in comparison with IR-SFLA and PPNGA. The method PPNGA presents instable performances which are reflected in relative good performance on $\mathrm{F}_{2}$ and $\mathrm{F}_{5}$, especially the $\mathrm{F}_{4}$ benchmarks and poor performance on $\mathrm{F}_{3}$.

From the MNIG and SR in the bottom of Table 1, the SR of IR-SFLA are all " $100 \%$ " which denotes IR-SFLA can obtain the final theoretical optimum of each benchmark in 20 independent simulations which verify the high reliability standard of IR-SFLA. Furthermore, the MNIG of IR-SFLA are the minimum within all the algorithms. However, from the observation of compared algorithms, although the IBCO can get the best results in $\mathrm{F}_{1}, \mathrm{~F}_{3}$, and $\mathrm{F}_{4}$, its $\mathrm{MNIG}$ are much bigger than that in IR-SFLA that indicates the lower convergence speed than the latter. The PPNGA performs well in $\mathrm{F}_{4}$ but is difficult to converge to optimum of other benchmarks. The rest of compared algorithms can not acquire the theoretical optimal solutions of each benchmark but perform better if the precision demand is relatively low. Therefore, the IRSFLA displays the remarkable advantages on reliability and convergence speed compared to others in terms of $F_{1}$ and $F_{5}$ benchmarks.

In terms of test ranks from Table 2, the less test result is, the better performance algorithm has. It can be seen that IR-SFLA is well positioned among all algorithms which denotes better performance and significant advantages on computational precision and reliability in comparison with other algorithms.

From the results summarized above, it can be concluded that the improvements for SFLA present efficient effects on overall search capability and keep a fast convergence speed compared with other rivals. To sum up, the IR-SFLA is more adaptive and effective in solving the optimization problem in continuous space.

\subsection{The IB-SFLA to Solve UC Subproblem}

3.4.1. Structure and Initialization of Frog Individual for UC. The frog individual of UC subproblem represents on or off
( 1 or 0$)$ state of the $k$ th unit at $j$ th interval. Therefore, the structure of individual for UC can be described as an integermatrix $U_{j, k}$ with value 0 or 1 and shown as follows:

$$
U_{j, k}=\left[\begin{array}{ccccc}
u_{1,1} & u_{2,1} & u_{3,1} & \cdots & u_{k, 1} \\
u_{1,2} & u_{2,2} & u_{3,2} & \cdots & u_{k, 2} \\
u_{1,3} & u_{2,3} & u_{3,3} & \cdots & u_{k, 3} \\
\vdots & \vdots & \vdots & \vdots & \vdots \\
u_{1, j} & u_{2, j} & u_{3, j} & \cdots & u_{k, j}
\end{array}\right]
$$

where $k$ and $j$ are the number of units and operation duration, respectively; the upper limit of $j$ is 24 , i.e., a day; $u_{k, j}$ represents the state of $k$ th unit at $j$ th interval.

$u_{k, j}$ is initialized with 0 or 1 at each period through following (23).

$$
u_{k, j}= \begin{cases}1 & \text { if } \operatorname{rand}()>0.5 \\ 0 & \text { otherwise }\end{cases}
$$

where $u_{k, j}$ is the same meaning with that in (22); $\operatorname{rand}()$ denotes random number between 0 and 1 .

\subsubsection{Strategies for Handling the Constraints}

(1) The Supplement and Repair Strategy for Unit Reserve Constraint. There are two cases during the process of optimal load distribution between each unit. Case 1 is that the total maximum load between all operating units $\sum_{i=1}^{k} u_{i} P_{i, \max }$ is less than the sum of requirement $N_{t \text {,plan }}$ and reserve capacity $P_{R}^{t}$; Case 2 is the total minimum load of all operating units; $\sum_{i=1}^{k} u_{i} P_{i, \min }$ is more than load requirement $N_{t, \text { plan }}$ and reserve capacity $P_{R}^{t}$.

In terms of the Cases mentioned above, a dynamic processing method based on priority list of unit startup and shutdown is proposed. The priority list is ranked in ascending order according to the average unit water consumption rate (AUWCR). The AUWCR calculation formula is shown in

$$
F_{\text {avg }}=\frac{\sum_{i=1}^{k}\left(Q_{i}\left(P_{i}\right) / P_{i}\right)}{k} \quad(i=1,2,3 \cdots, k)
$$

$F_{\text {avg }}$ denotes the average rate of water consumption; $P_{i}$ is the distributed power load within stable operation region (SOR); $Q_{i}\left(P_{i}\right)$ represents the corresponding water consumption; $k$ is maximum power load within SOR, setting $1 \mathrm{MW}$ as an interval.

If Case 1 is activated, turn up the current offline units satisfying the minimum down time constraint in turn according to ascending order in the priority list until the reserve capacity constraint is satisfied. Otherwise, shut down the online units satisfying the minimum operation time constraint in turn according to descending order in the priority list until the reserve capacity constraint is satisfied.

(2) The Strategy for Repairing Minimum Up and Down Time Constraint. To satisfy the minimum up and down 
TABLE 2: The ranks obtained by nonparametric test of Friedman and Kruskal-Wallis for IR-SFLA and compared algorithms.

\begin{tabular}{|c|c|c|c|c|c|c|}
\hline \multirow{2}{*}{ Nonparametric Test } & \multicolumn{6}{|c|}{ Algorithms } \\
\hline & PPNGA & EACO & SFLA & CGSA & IBCO & IR-SFLA \\
\hline Friedman (Rank) & 3.42 & 4.17 & 3.96 & 5.02 & 3.00 & 1.45 \\
\hline Kruskal-Wallis (Rank) & 58.94 & 73.235 & 69.865 & 91.13 & 50.43 & 19.4 \\
\hline
\end{tabular}

Best results are marked in bold.

time constraint, a repair strategy is conducted to adjust the units on and off state. The variables Opentime $(i, j, k)$ and Closetime $(i, j, k)$ are introduced to record startup and shutdown duration of each unit attached to $i$ th frog at $j$ th period. The record process is described in (25). Meanwhile, the startup and shutdown durations stored in Opentime $(i, j, k)$ and Closetime $(i, j, k)$ are verified whether the minimum up and down time constraint is satisfied or not. If not, unit state remains unchanged; otherwise, the frog can realize the state transition in line with (15).

$$
\begin{aligned}
& \text { Opentime }[i, j, k] \\
& = \begin{cases}\text { Opentime }[i, j-1, k]+1 & \text { if } u[i, j, k]=1 \\
0 & \text { if } u[i, j, k]=0\end{cases}
\end{aligned}
$$

$$
\begin{aligned}
& \text { Closetime }[i, j, k] \\
& = \begin{cases}\text { Closetime }[i, j-1, k]+1 & \text { if } u[i, j, k]=0 \\
0 & \text { if } u[i, j, k]=1\end{cases}
\end{aligned}
$$

3.5. The IR-SFLA to Solve ELD Subproblem Based on Optimal Economic Operation Table. A wise and feasible unit commitment is acquired with handling the UC subproblem, and proposed IR-SFLA is applied to realize highly efficient load distribution between committed units.

3.5.1. Structure and Initialization of ELD. The structure of ELD is correlated with that in UC, which consists of power output for all committed hydropower units. The specific structure is illustrated as follows:

$$
P_{j, k}=\left[\begin{array}{ccccc}
u_{1,1} P_{1,1} & u_{2,1} P_{2,1} & u_{3,1} P_{3,1} & \cdots & u_{k, 1} P_{k, 1} \\
u_{1,2} P_{1,2} & u_{2,2} P_{2,2} & u_{3,2} P_{3,2} & \cdots & u_{k, 2} P_{k, 2} \\
u_{1,3} P_{1,3} & u_{2,3} P_{2,3} & u_{3,3} P_{3,3} & \cdots & u_{k, 3} P_{k, 3} \\
\vdots & \vdots & \vdots & & \vdots \\
u_{1, j} P_{1, j} & u_{2, j} P_{2, j} & u_{3, j} P_{3, j} & \cdots & u_{k, j} P_{k, j}
\end{array}\right]
$$

where $P_{k, j}$ represents the power output for the $k$ th unit at $j$ th interval; the meaning of $u_{k, j}$ is in line with that in UC structure.

Different with UC initialization, the ELD is real-coded continuity optimization problem. Therefore, initialization process of ELD is according to method based on chaotic theory. The logistic mapping equation is in line with (17); however, the reflection equation is demonstrated as follows with corresponding changes according to (18).

$$
P_{j, k}=\frac{\left(x_{j}^{t}+1\right)}{2} *\left(P_{k, \text { max }}-P_{k, \text { min }}\right)+P_{k, \text { min }}
$$

$x_{j}^{t}$ denotes the chaotic variable generated by improved logistic mapping formula; $P_{k, \text { min }}$ and $P_{k \text {,max }}$ are lower and upper limit for $k$ th unit.

3.5.2. The Optimal Economic Operation Table. The economic operation for large scale hydropower station, especially in terms of Three Gorges hydropower station, is multidimensional, complex nonlinear optimization problem. In order to simplify the solving process and significantly improve the computation efficiency, the optimal economic operation table is established by the proposed IR-SFLA. The table formulation is on premise that all power units are involved in power generation; i.e., all units are startup. In addition, the SOR of units is taken into consideration to keep unit operated in high efficiency and safe region. The established economic operation table is stored in database, thus making it easier to call the table. The solving formula is demonstrated as follows:

$$
\begin{aligned}
N_{1}, Q_{1}\left(N_{1}, H\right) & =0 \\
N_{i} & =N_{i}+P_{i, j} \\
Q_{i}\left(N_{i}, H\right) & =Q_{i}\left(N_{i}, H\right)+q_{i, j} \\
\text { Fitbest }\left(Q_{i}\left(N_{i}, H\right)\right) & =\min \left(Q_{i}\left(N_{i}, H\right)\right)
\end{aligned}
$$

$N_{1}, Q_{1}\left(N_{1}, H\right)=0$ are boundary conditions in the initial stage; $N_{i}$ is accumulative load of units; $Q_{i}\left(N_{i}, H\right)$ denotes water consumption of power generation when total load and water head are $N_{i}$ and $H$, respectively; Fitbest $\left(Q_{i}\left(N_{i}, H\right)\right)$ is the best frog individual.

3.5.3. The Process of Invoking Optimal Economic Operation Table. As mentioned above, the optimal economic operation table is stored in database so as to be conveniently invoked. The number of startup units is recorded using the variable $m$ when a unit commitment is obtained by the UC submodule. The variable $l$ is defined as a count variable to locate the $l$ th line in optimal economic operation table. The load distribution between units is obtained by locating the specified line in optimal economic operation table according to the load demand $N_{t, \text { plan }}$ corresponding to each interval. The linear interpolation method is adopted to acquire the load distribution if it cannot 


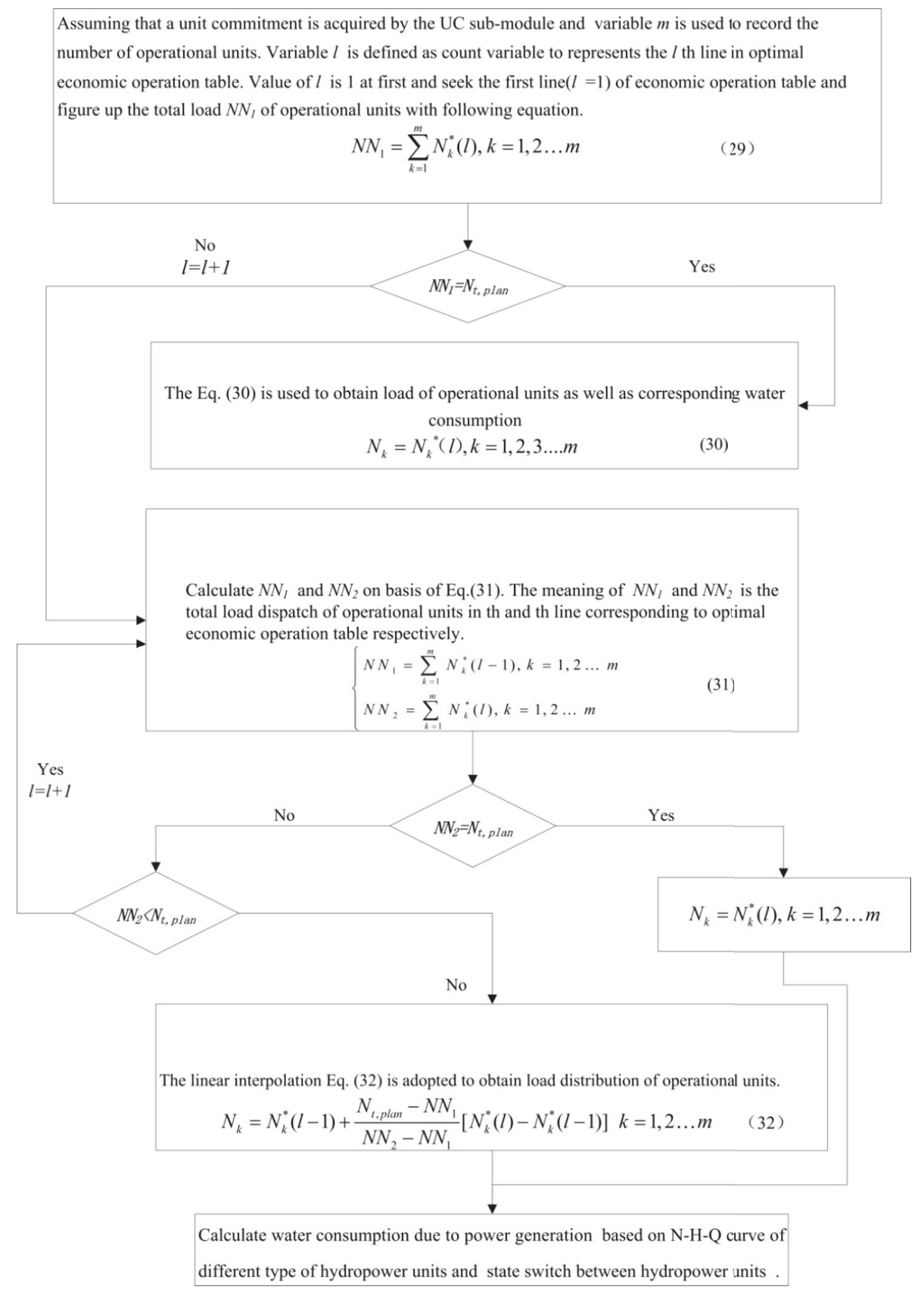

FIGURE 6: The flowchart of invoking optimal economic operation table.

be located directly in the table. The flowchart of invoking optimal economic operation table is demonstrated in Figure 6 and the detailed procedures are shown as follows.

Step 1. Seek the first line $(l=1)$ of economic operation table and figure up the total load $N N_{1}$ of $m$ operational units according to (29). Compare $N N_{1}$ with load demand and judge whether it is submitted to $N N_{1}=N_{t, \text { plan }}$ or not. If not, $l=l+1$ and turn to Step 2. Otherwise, terminate the search and (30) is adopted to obtain the load distribution of operational units and corresponding water consumption.

$$
\begin{aligned}
N N_{1} & =\sum_{k=1}^{m} N_{k}^{*}(l), \quad k=1,2 \ldots m \\
N_{k} & =N_{k}^{*}(l), \quad k=1,2,3 \ldots m
\end{aligned}
$$

$N_{k}^{*}(l)$ denotes load distribution of $k$ th operational unit in $l$ th line of optimal economic operation table; $N N_{1}$ is total load distribution of all the operational units in $l$ th line. $N_{k}$ is intermediate variable to store $N_{k}^{*}(l)$.

Step 2. Calculate $N N_{1}$ and $N N_{2}$ in line with (31). $N N_{1}$, $\mathrm{NN}_{2}$ are the total load distribution of operational units 


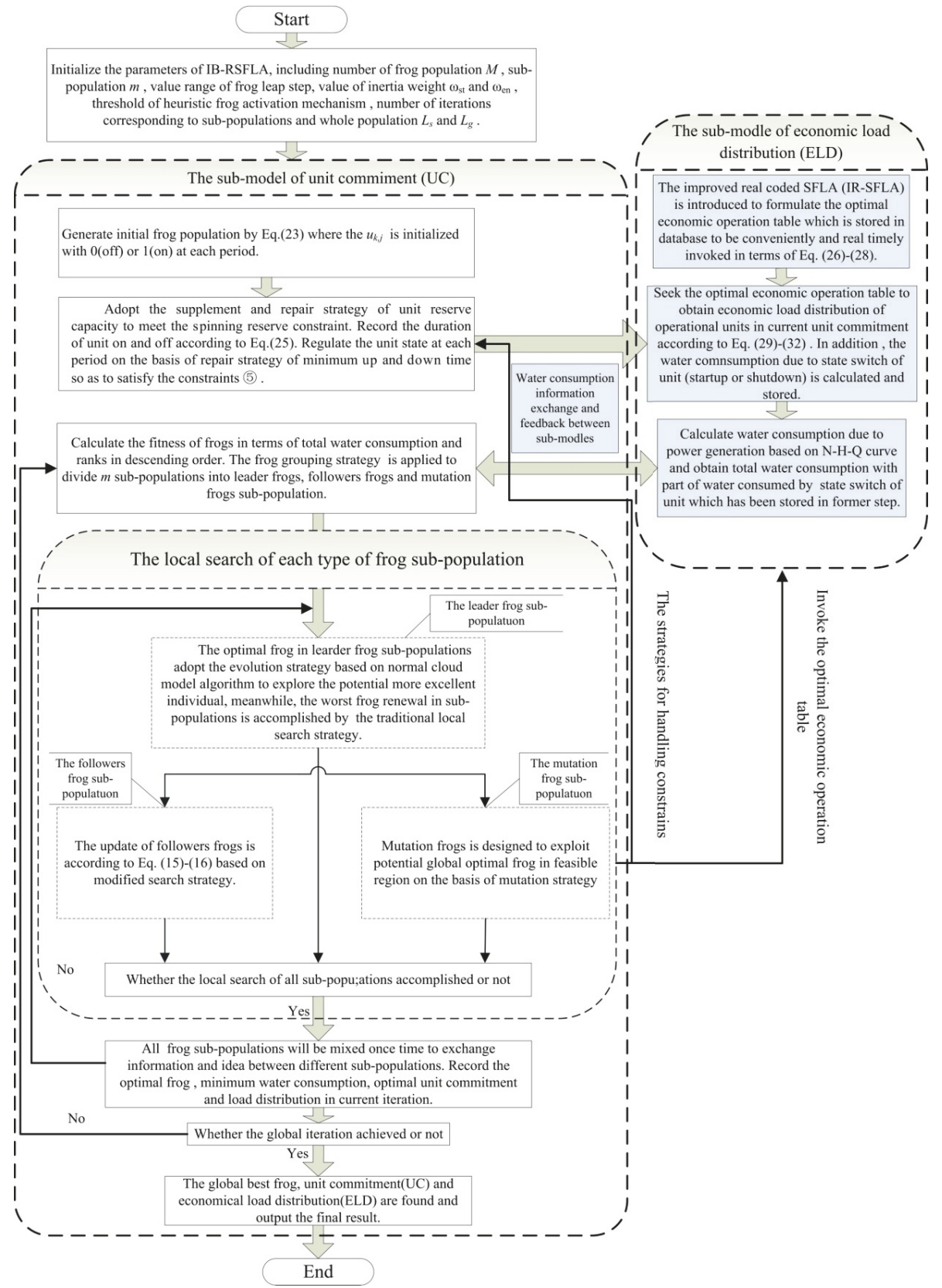

FIGURE 7: The flowchart of coupled external and internal model based on IBR-SFLA for solving STHGS.

corresponding to $l-1$ th and $l$ th lines in optimal economic operation table. Then go to Step 3.

$$
\begin{aligned}
& N N_{1}=\sum_{k=1}^{m} N_{k}^{*}(l-1), \quad k=1,2 \ldots m \\
& N N_{2}=\sum_{k=1}^{m} N_{k}^{*}(l), \quad k=1,2 \ldots m
\end{aligned}
$$

Step 3. Adjust $l$ according to $l=l+1$ and turn to Step 2 if the criterion $\mathrm{NN}_{2}<N_{t, \text { plan }}$ is satisfied. Otherwise, continue to judge whether the criterion $N N_{2}=N_{t, \text { plan }}$ is satisfied; if it is, the power load of each operational unit is obtained by $N_{k}=$
$N_{k}{ }^{*}(l), k=1,2,3 \ldots . m$. Otherwise, the linear interpolation of (32) is applied to obtain load distribution of operational units.

$$
\begin{aligned}
& N_{k}=N_{k}^{*}(l-1) \\
& \quad+\frac{N_{t, p l a n}-N N_{1}}{N N_{2}-N N_{1}}\left[N_{k}^{*}(l)-N_{k}^{*}(l-1)\right] \\
& \quad k=1,2 \ldots m
\end{aligned}
$$

Step 4. Calculate water consumed by power generation and startup and shutdown transition between hydropower units based on NHQ curve. 


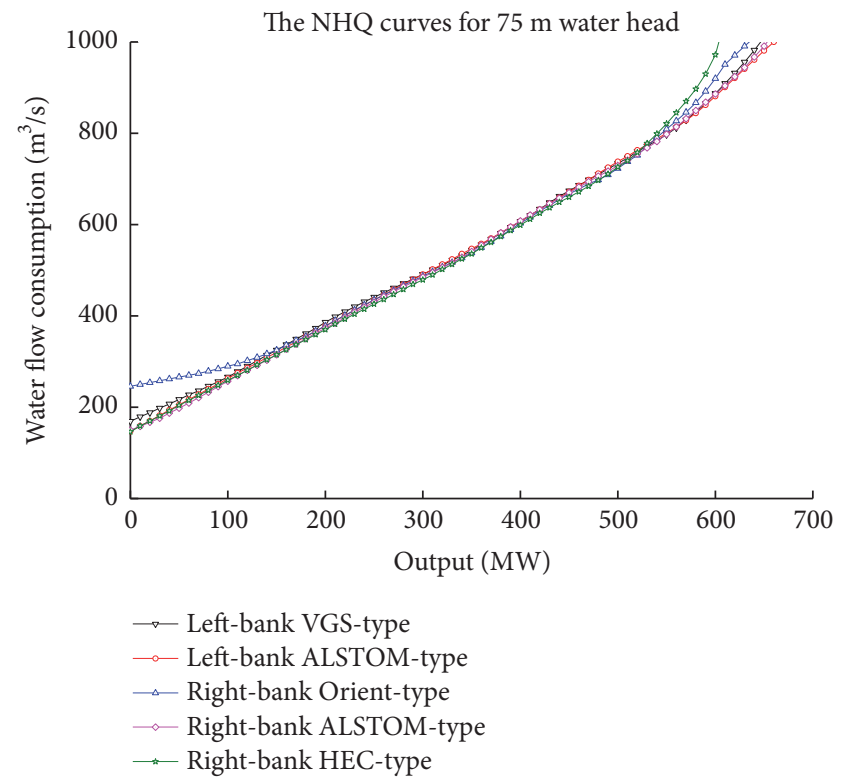

(a) $75 \mathrm{~m}$

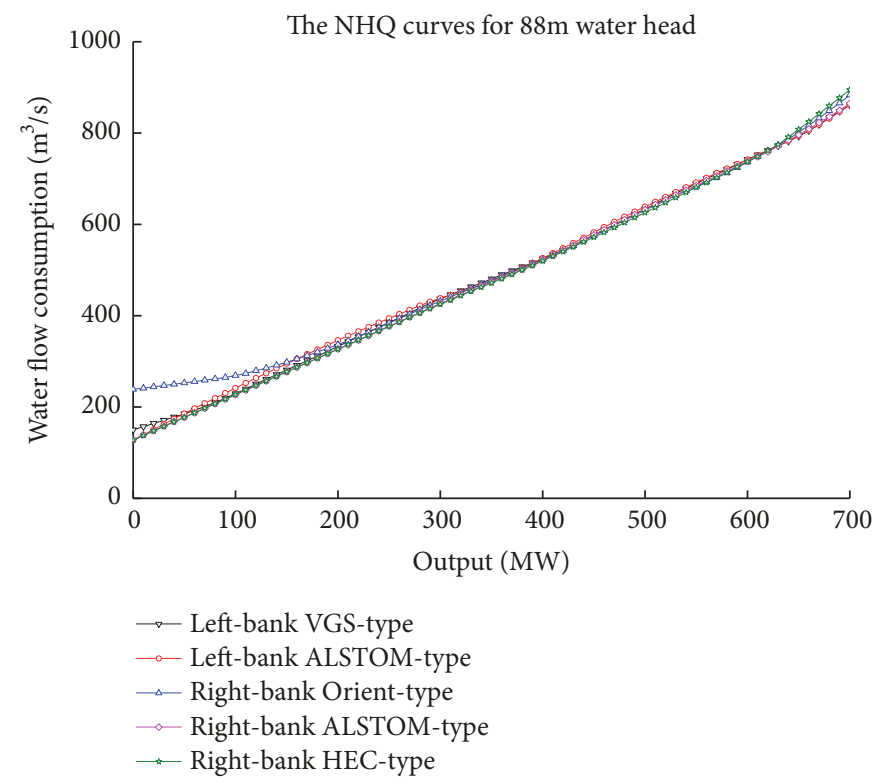

(b) $88 \mathrm{~m}$

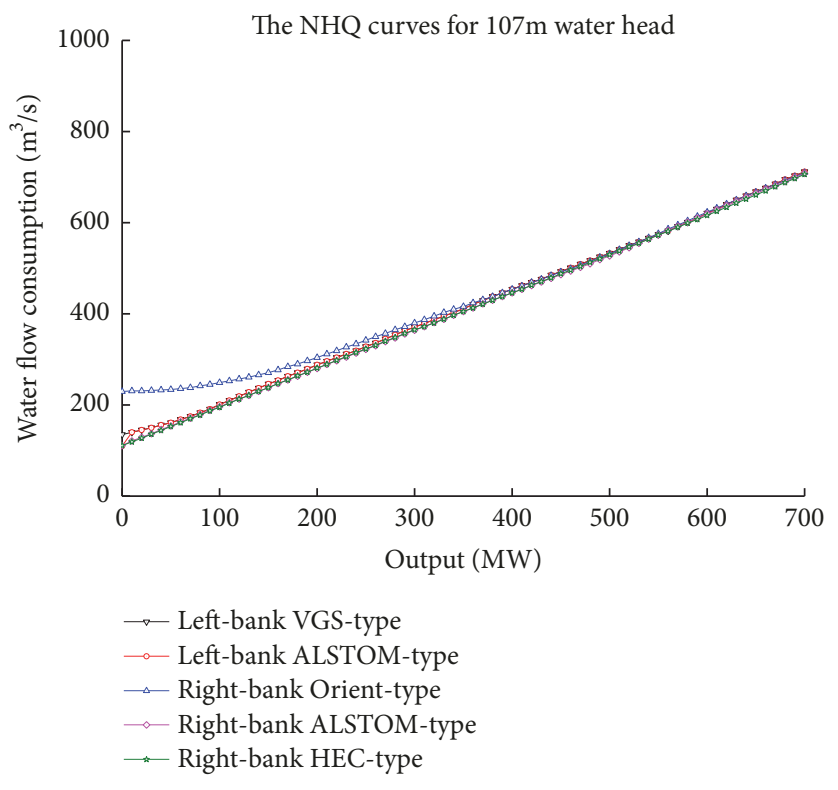

(c) $107 \mathrm{~m}$

Figure 8: The NHQ curve of left- and right-bank hydro units corresponding to $75 \mathrm{~m}, 88 \mathrm{~m}$, and $107 \mathrm{~m}$ water head.

3.6. The Coupled External and Internal Model for STHGS Problem. The STHGS problem consists of the unit commitment (UC) and load distribution subproblems. Therefore, the coupling model consists of the UC (external submodel) and ELD (internal submodel). The UC submodel delivers message to the ELD submodel in order to realize optimal load distribution and compute total water consumption. On the contrary, the information about water consumption due to power generation and unit startup and shutdown pass back to the UC submodel. The specific steps are listed as follows.

Step 1. Initialize the parameters of IBR-SFLA, including number of frog populations $M$, subpopulations $m$, range of frog leap step, inertia weights $\omega_{\text {st }}$ and $\omega_{\text {en }}$, threshold of heuristic frog activation mechanism, iterations in subpopulations, and whole populations $L_{s}$ and $L_{g}$. 
Step 2. Generate initial frog population by (23) where $u_{k, j}$ is initialized with 0 (shutdown) or 1 (startup) at each period using the rand( ) function.

Step 3. Adopt the supplement and repair strategy of unit reserve capacity to satisfy the sinning reserve constraints. Record the duration of unit startup and shutdown according to (25). Regulate the unit state at each period with the repair strategy of minimum startup and shutdown time in order to satisfy constraint (5).

Step 4. Invoke and seek the optimal economic operation table to obtain load distribution of operational units and total water consumption including electricity generation and startup and shutdown of units. Calculate fitness of frogs which are ranked in descending order.

Step 5. The frog grouping strategy is applied to divide population into leader frogs, followers frogs, and mutation frogs subpopulations.

Step 6. The leader frogs subpopulation adopts the elite evolution strategy to search and update optimal frog in subpopulation while the worst frog renewal in subpopulations is conducted through traditional frog local search strategy; mutation frogs are designed to exploit potential global optimal frog in feasible region on the basis of mutation strategy; the followers frogs update is based on modified search strategy. The process of handling constraints is still according to strategies in Step 3. Then, finish the load distribution through the optimal economic operation table mentioned in Step 4. All subpopulations belonging to each type of frog are updated with corresponding strategy mentioned and renew the local best frog $u_{b}$ and global best $u_{g}$ in each iteration.

Judge whether the iteration of local search is up to the number preset or not; if it is, turn to Step 7. Otherwise, go back to Step 6 and continue to search until the local update is finished in all subpopulations.

Step 7. All subpopulations will be mixed to exchange information and idea between different subpopulations. Record the best frog, minimum water consumption, and optimal unit commitment and load distribution in current global shuffling iteration.

Judge whether the global shuffling iteration is achieved or not; if not, turn to Step 5. If it is, the global best frog, unit commitment, and load distribution are found and output the final results.

The flowchart of coupling external and internal model for STHGS is demonstrated in Figure 7.

\section{Case Study}

4.1. Three Gorges Hydropower Station Description. The Three Gorges hydropower station, the largest hydropower plant in the world, is located on the Yangtze River in China. The hydropower station is initially equipped with 26 mixed-flow hydro units of $700 \mathrm{MW}$, and 2 power supply hydro units of 50

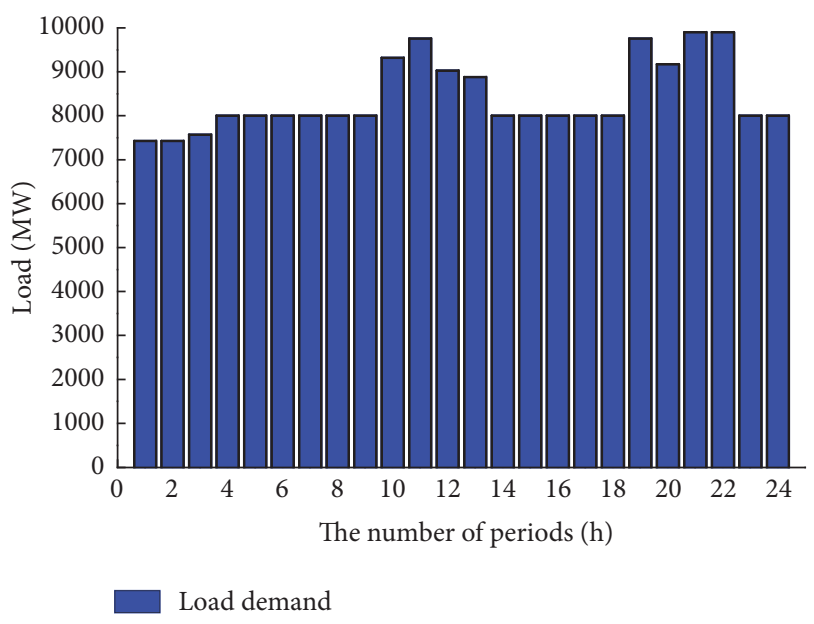

FIGURE 9: The daily load curves at each interval for hydropower station.

MW and 6 hydro units with 700MW installed capacity are equipped afterwards. Therefore, the total installed capacity of hydropower station is up to $22,500 \mathrm{MW}$. In this paper, the initial 26 hydro units containing 6 left-bank VGS units, 8 leftbank ALSTOM units, 4 right-bank ORIENT power units, 4 right-bank ALSTOM units, and 4 right-bank HEC units are selected as simulation operation objects.

In this study, the $75 \mathrm{~m}, 88 \mathrm{~m}$, and $107 \mathrm{~m}$ water head are selected as three simulation cases and scheduling periods are 24 hours. The stable operation regions (SOZ) of generating units under $75 \mathrm{~m}, 88 \mathrm{~m}$, and $107 \mathrm{~m}$ water head are listed in Table 3. Meanwhile, the flow characteristic curves (NHQ curves) of hydro units corresponding to $75 \mathrm{~m}, 88 \mathrm{~m}$, and $107 \mathrm{~m}$ water head are demonstrated in Figure 8. The daily load demands at each interval for hydropower station are drawn in Figure 9.

4.2. Parameter Settings. The parameter settings significantly affect the efficiency of the IB-RSFLA including search ability, stability, and computation speed. In this study, we set a group of parameter combinations and make comparison of simulation results to find the best control parameter combination. The optimal control parameter settings are described as follows:

The number of frog populations $M$ is 80 ; the number of subpopulations $m$ is 10 ; the number of frogs in each subpopulation $h$ is 8 ; the frog leap step is valued in the interval $[-4,4]$; the value of inertia weights $\omega_{s t}$ and $\omega_{e n}$ are, respectively, 0.9 and 0.4 ; the iterations number of local search in subpopulation $L_{s}$ is 10 and global search $L_{g}$ is 50 . In addition, the similarity threshold $\varepsilon$ used to motivate the heuristic frog activation mechanism is tested to value $10^{-3}$.

4.3. Results and Analysis. The $75 \mathrm{~m}, 88 \mathrm{~m}$, and $107 \mathrm{~m}$ water heads are selected as the cases and 20 independent simulations are conducted to find the best solution. The unit commitment and economic load dispatch corresponding to the best solution under $75 \mathrm{~m}, 88 \mathrm{~m}$, and $107 \mathrm{~m}$ water head 

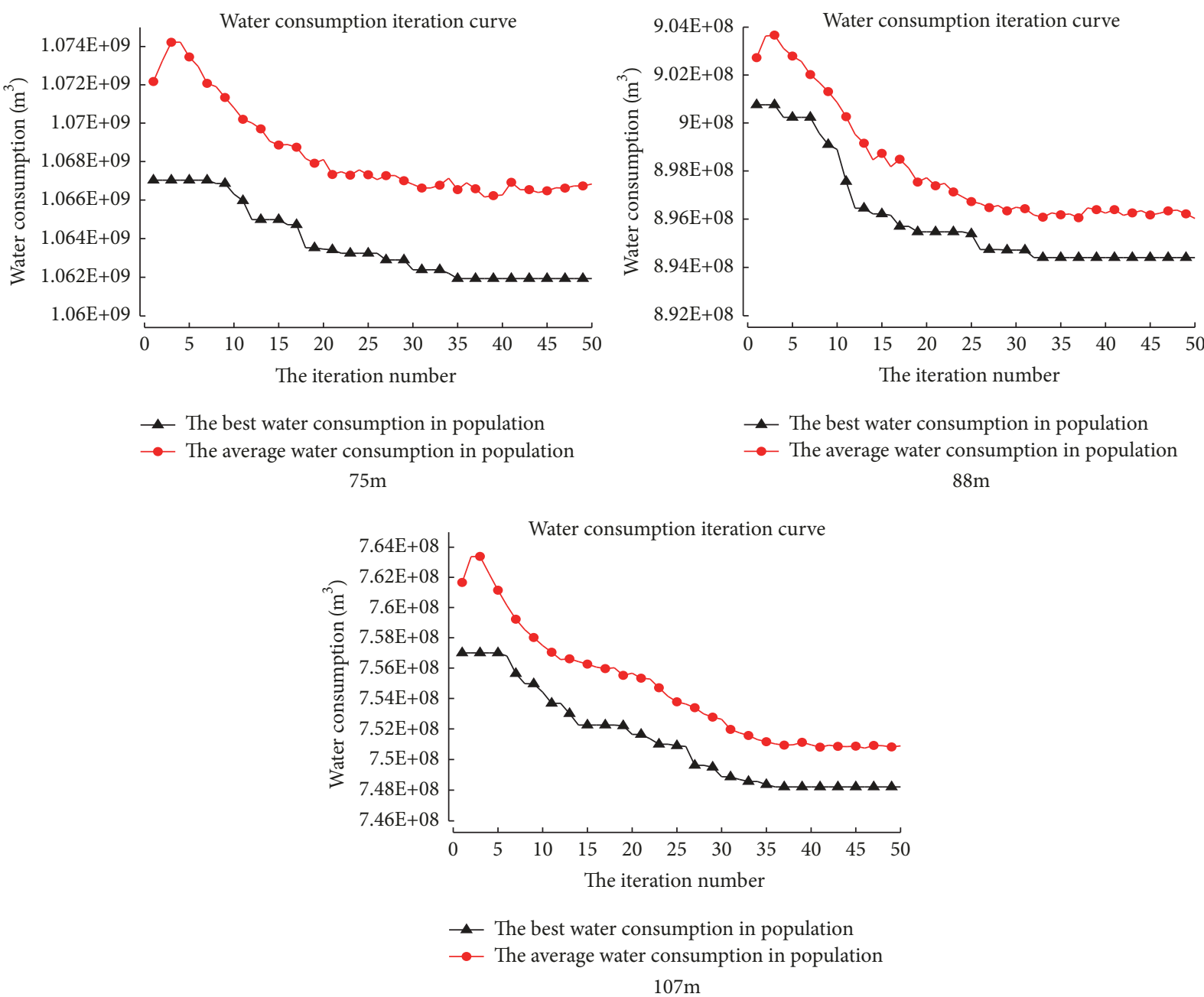

FIGURE 10: The iterative curves of water consumption at each generation corresponding to $75 \mathrm{~m}, 88 \mathrm{~m}$, and $107 \mathrm{~m}$ water head.

TABLE 3: Stable operation region of each unit in Three Gorges corresponding to $75 \mathrm{~m}, 88 \mathrm{~m}$, and $107 \mathrm{~m}$ water head (Unit: MW).

\begin{tabular}{lccccc}
\hline $\begin{array}{l}\text { Water } \\
\text { head }\end{array}$ & $\begin{array}{c}\text { Left-bank } \\
\text { VGS units } \\
(1-6 \#)\end{array}$ & $\begin{array}{c}\text { Left-bank } \\
\text { ALSTOM units } \\
(7-14 \#)\end{array}$ & $\begin{array}{c}\text { Right-bank } \\
\text { ORIENT power units } \\
(15-18 \#)\end{array}$ & $\begin{array}{c}\text { Right-bank } \\
\text { ALSTOM units } \\
(19-22 \#)\end{array}$ & $\begin{array}{c}\text { Right-bank } \\
\text { HEC units } \\
(23-26 \#)\end{array}$ \\
\hline $75 \mathrm{~m}$ & $410 \sim 645$ & $435 \sim 640$ & $390 \sim 615$ & $430 \sim 665$ & $385 \sim 575$ \\
$88 \mathrm{~m}$ & $475 \sim 700$ & $525 \sim 700$ & $470 \sim 700$ & $500 \sim 700$ & $465 \sim 700$ \\
$107 \mathrm{~m}$ & $570 \sim 700$ & $615 \sim 700$ & $585 \sim 700$ & $595 \sim 700$ & $585 \sim 700$ \\
\hline
\end{tabular}

are demonstrated in Table 4, respectively. In inspection of economic load dispatch in Table 4, all hydropower units are operated under stable operation region at each interval, and the hydropower unit output limits (3) and prohibited operating region constraint (4) are rigidly satisfied. Furthermore, Table 4 shows the corresponding unit commitment (UC) strictly satisfies minimum up and down time constraint, which illustrate the state of units (startup and shutdown) is reasonably arranged using "strategies for handling the constraints" in Section 3.4.2. The effective UC contributes to avoiding units startup and shutdown frequently and prolonging the service life of hydropower units.
The iterative curves of best and average water consumption at each generation under 75,88 , and $107 \mathrm{~m}$ water head are shown in Figure 10. Furthermore, a bit of "fluctuant points" in the curves denote the possible local optimal point. Before "fluctuant points", the search by IBR-SFLA is close to standstill state which indicates algorithm trapped in local optimum. However, the search is reactivated and explored further to gradually converge to best solution after "fluctuant points". The reactivation can be attributed to evolution operator, modified search strategy of followers frog proposed in IBR-SFLA which contribute algorithm to effectively escape from local optimum and motivate overall search capability. 


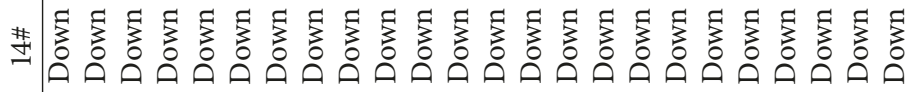

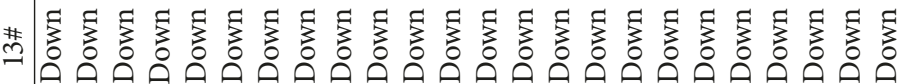

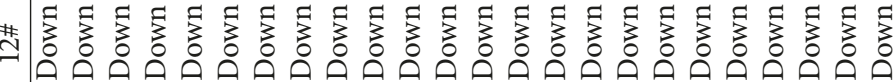

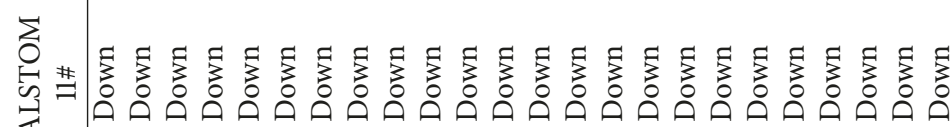

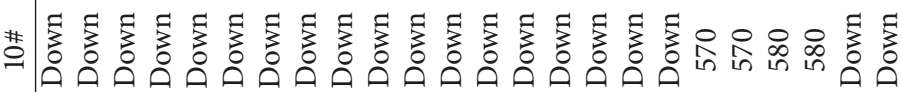
空

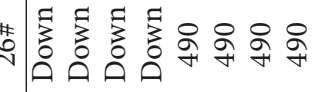

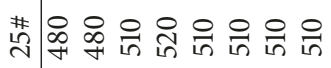

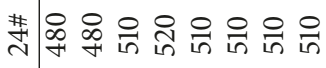

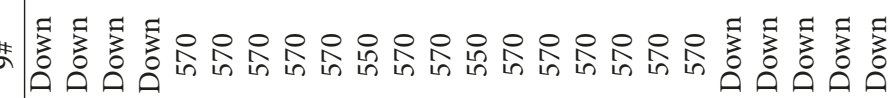

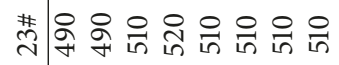

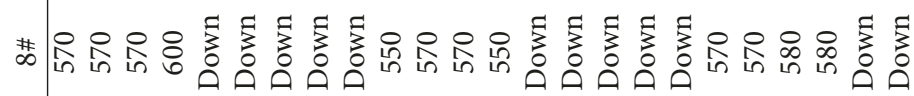

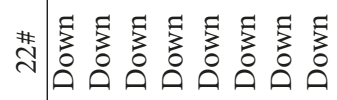

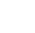

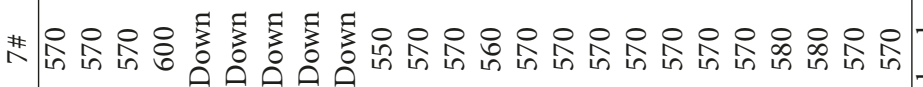

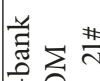

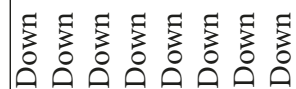

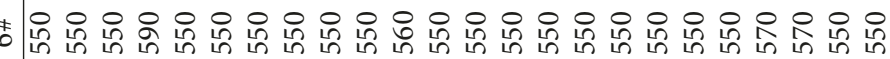

舟年

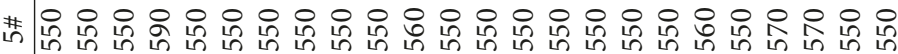

* s

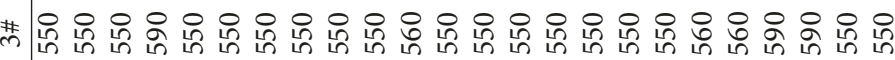

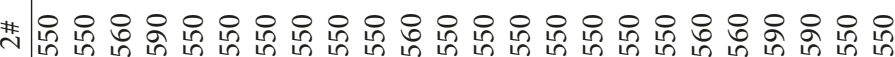

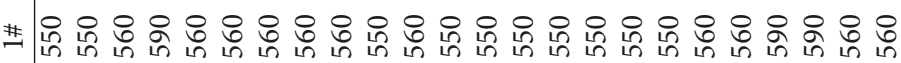

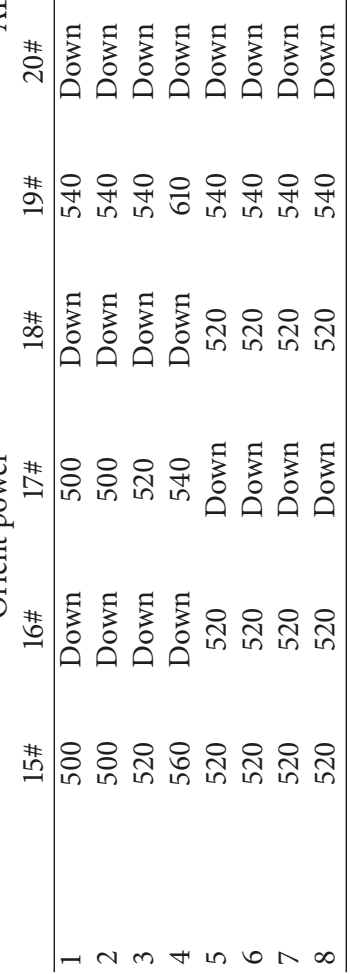




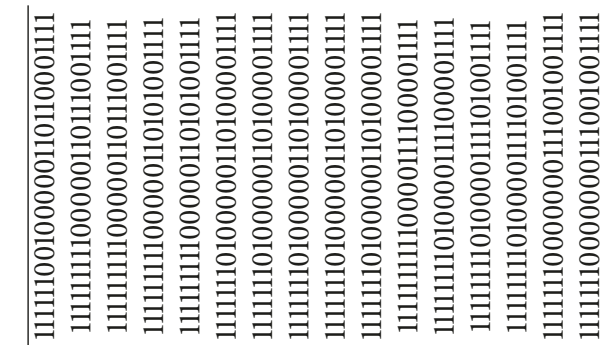

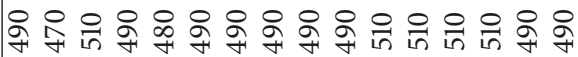

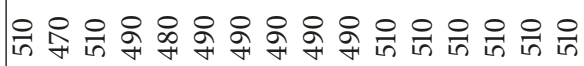

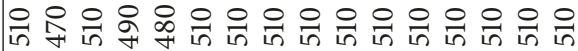

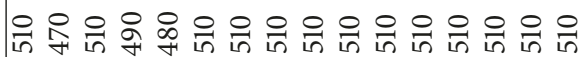

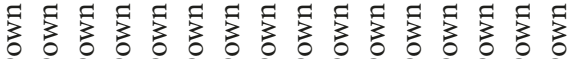
苞

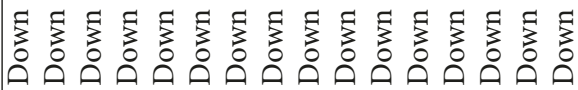

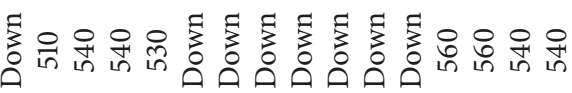

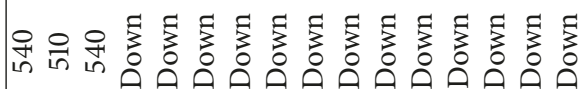

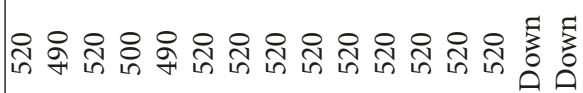

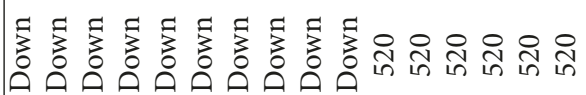

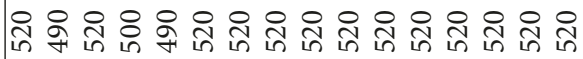

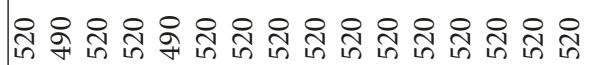

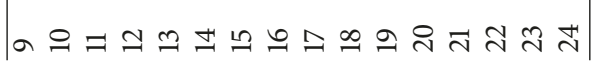

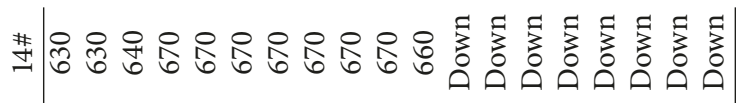

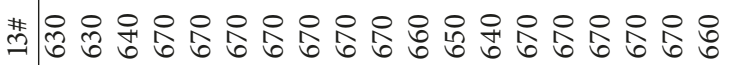

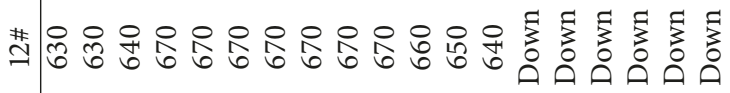

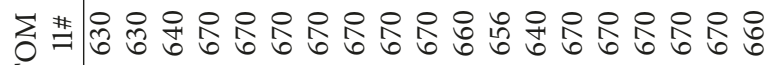
占

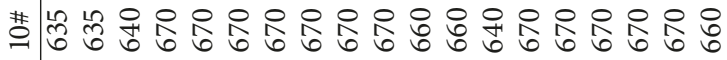

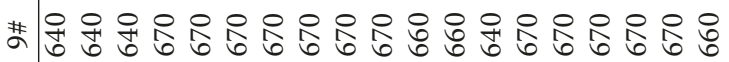

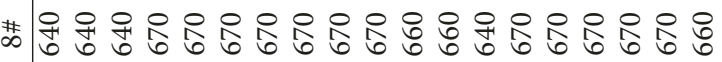

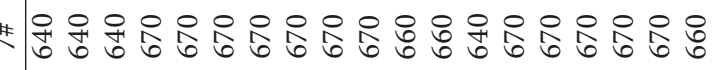

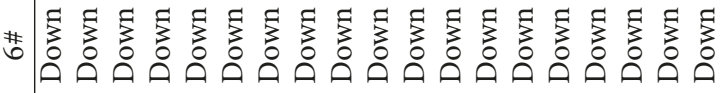

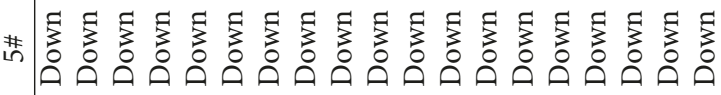

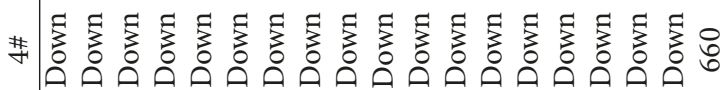
乌

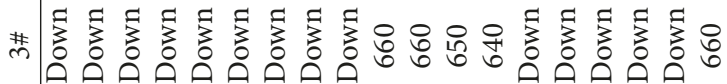

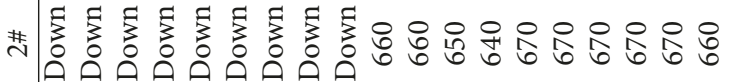

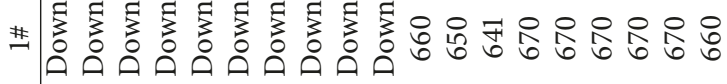

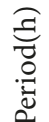

ー 


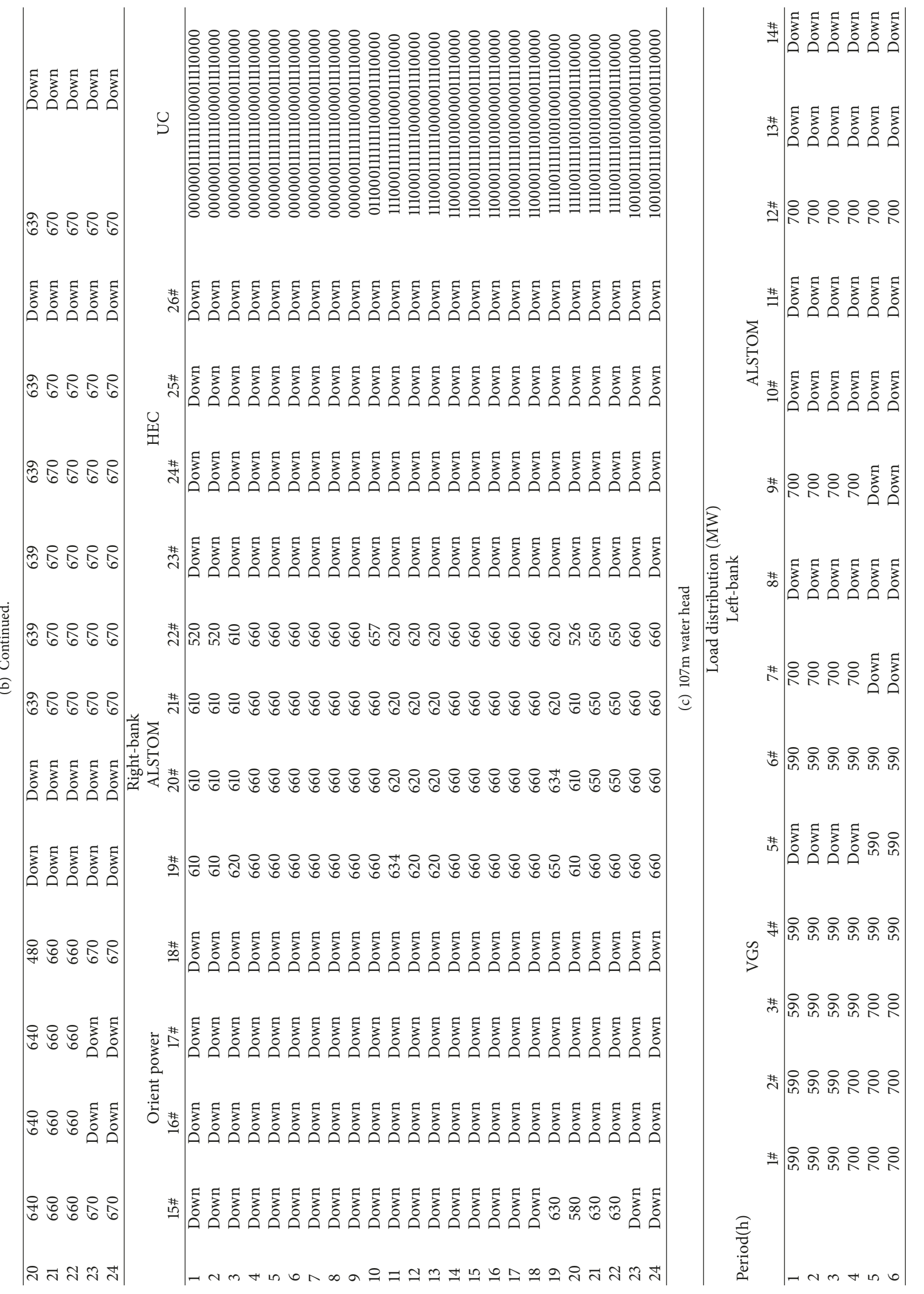




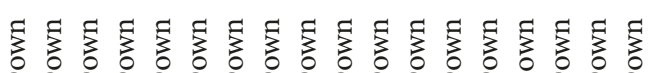
3848888888848888

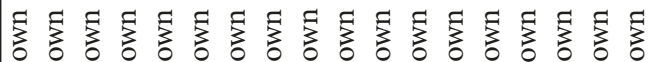

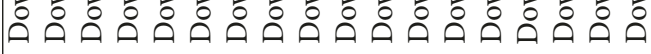

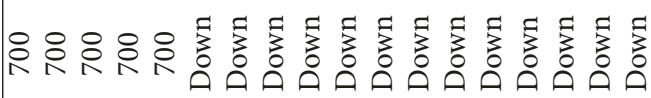

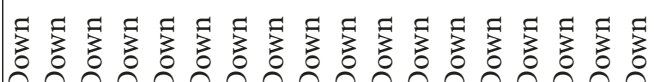

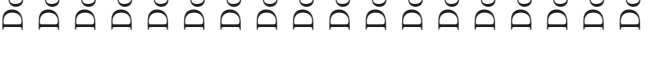

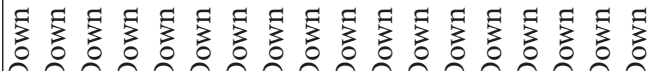

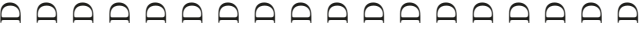

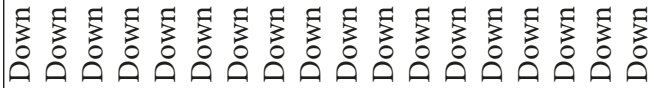

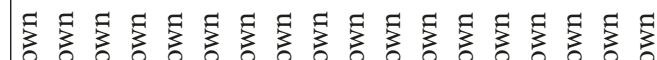
30

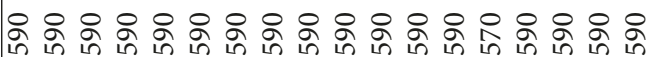

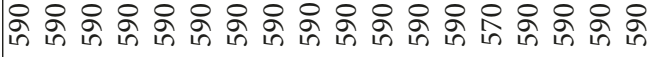

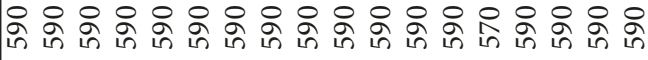

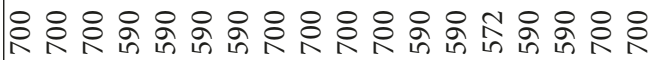

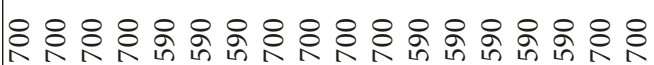

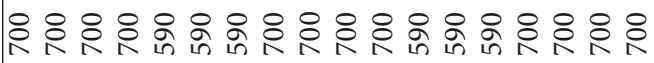

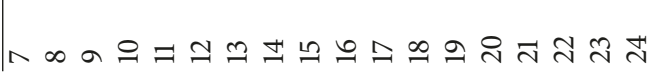

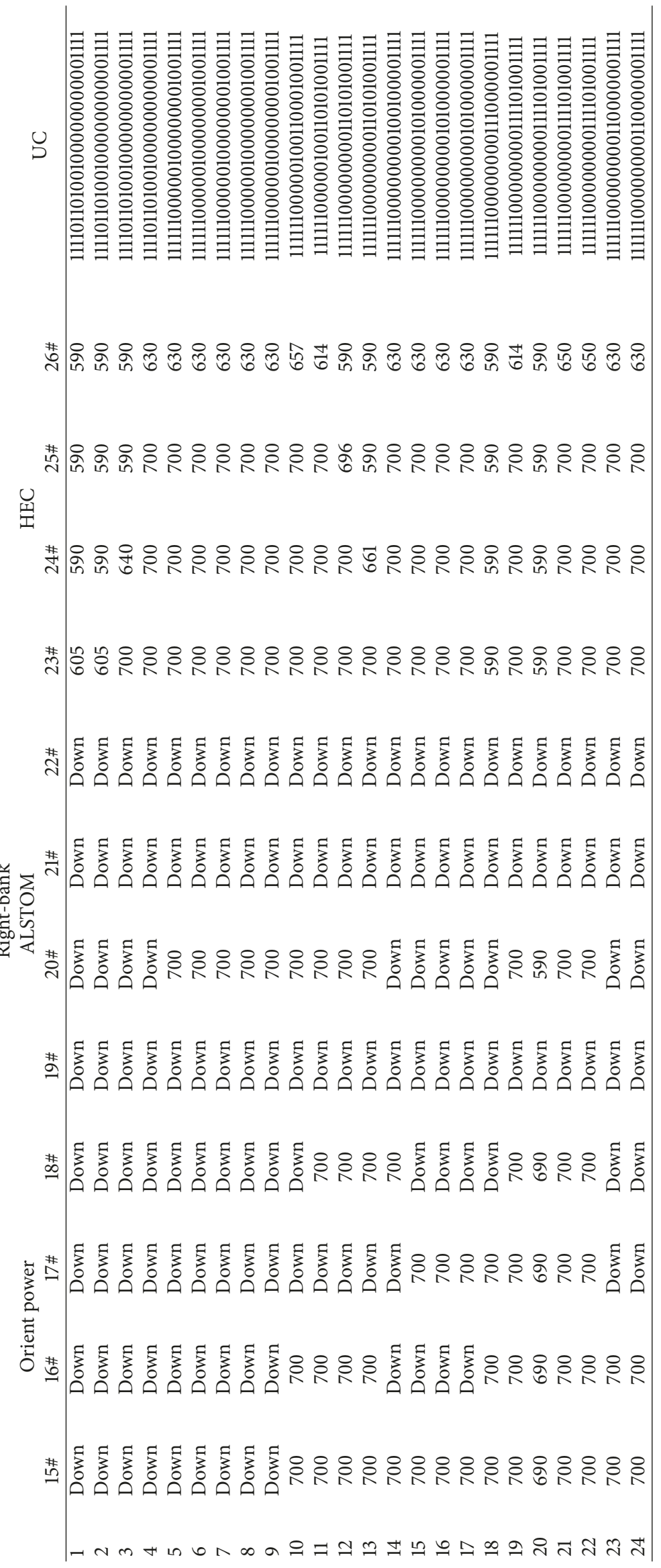




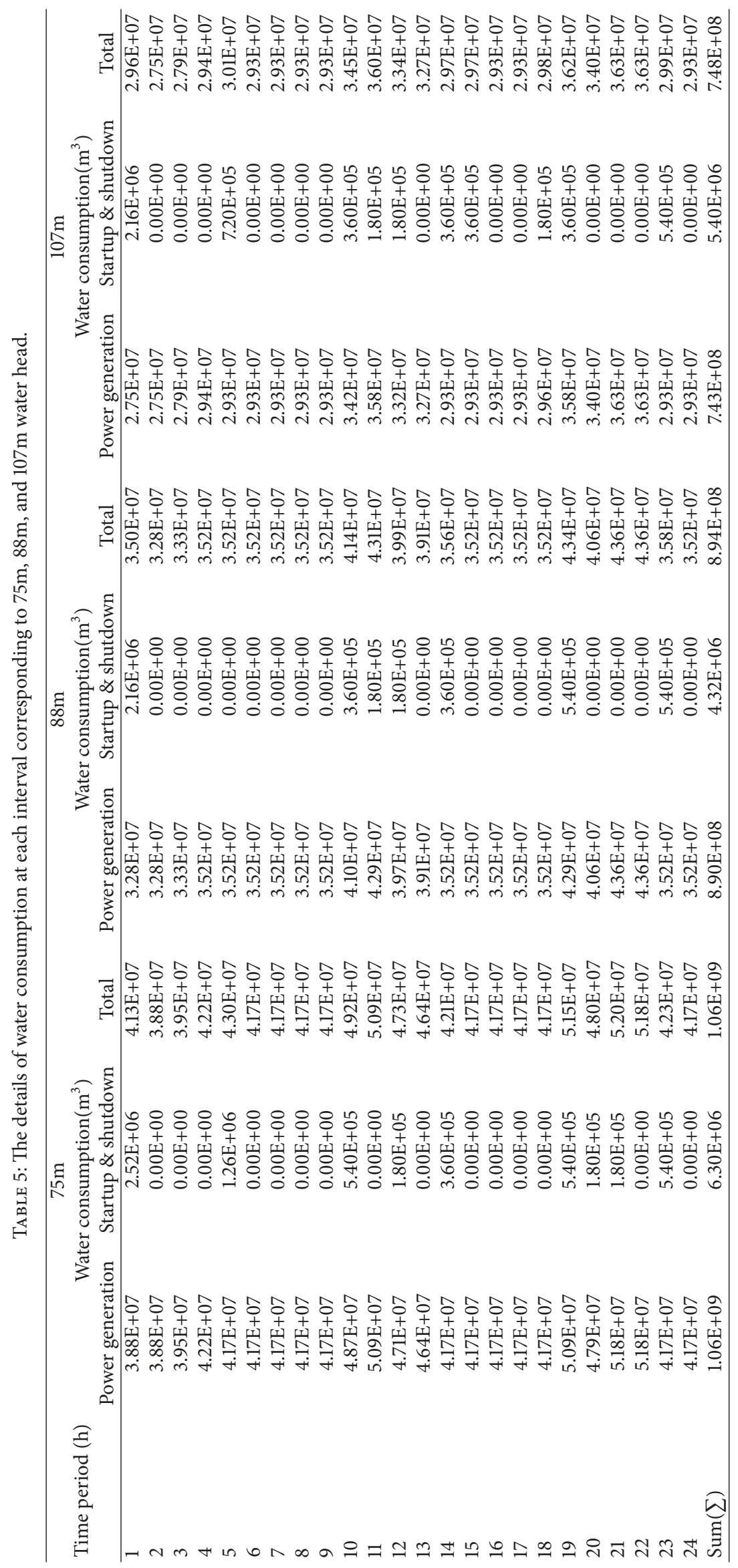


TABLE 6: The water consumption and average computational time of each algorithm corresponding to 75,88 , and $107 \mathrm{~m}$ water head.

\begin{tabular}{|c|c|c|c|c|c|}
\hline \multirow[t]{2}{*}{$\begin{array}{l}\text { Water } \\
\text { head }\end{array}$} & \multirow[t]{2}{*}{ Algorithm } & \multicolumn{2}{|c|}{$\begin{array}{l}\text { Water consumption } \\
\qquad\left(10^{8} \mathrm{~m}^{3}\right)\end{array}$} & \multirow[t]{2}{*}{$\begin{array}{l}\text { Standard deviation } \\
\text { (STD) }\end{array}$} & \multirow[t]{2}{*}{ Simulation time (s) } \\
\hline & & Mean & Optimal & & \\
\hline \multirow{6}{*}{$75 \mathrm{~m}$} & LAGA & $1.074 \mathrm{E}+01$ & $1.073 \mathrm{E}+01$ & 8.90E-03 & 224 \\
\hline & EACO & $1.068 \mathrm{E}+01$ & $1.067 \mathrm{E}+01$ & $8.58 \mathrm{E}-03$ & 105 \\
\hline & CGSA & $1.067 \mathrm{E}+01$ & $1.065 \mathrm{E}+01$ & $1.20 \mathrm{E}-02$ & 93 \\
\hline & FA & $1.066 \mathrm{E}+01$ & $1.065 \mathrm{E}+01$ & $9.50 \mathrm{E}-03$ & 76 \\
\hline & IBCO & $1.066 \mathrm{E}+01$ & $1.064 \mathrm{E}+01$ & $8.13 \mathrm{E}-03$ & 82 \\
\hline & IBR-SFLA & $1.063 \mathrm{E}+01$ & $1.062 \mathrm{E}+01$ & $4.46 \mathrm{E}-03$ & 64 \\
\hline \multirow{6}{*}{$88 \mathrm{~m}$} & LAGA & $9.071 \mathrm{E}+00$ & $9.057 \mathrm{E}+00$ & $5.60 \mathrm{E}-03$ & 218 \\
\hline & EACO & $9.006 \mathrm{E}+00$ & $8.994 \mathrm{E}+00$ & $8.68 \mathrm{E}-03$ & 100 \\
\hline & CGSA & $9.000 \mathrm{E}+00$ & $8.984 \mathrm{E}+00$ & $9.14 \mathrm{E}-03$ & 92 \\
\hline & FA & $8.984 \mathrm{E}+00$ & $8.971 \mathrm{E}+00$ & $6.93 \mathrm{E}-03$ & 75 \\
\hline & IBCO & $8.992 \mathrm{E}+00$ & $8.978 \mathrm{E}+00$ & $8.08 \mathrm{E}-03$ & 79 \\
\hline & IBR-SFLA & $8.950 \mathrm{E}+00$ & $8.944 \mathrm{E}+00$ & $3.49 \mathrm{E}-03$ & 63 \\
\hline \multirow{6}{*}{$107 \mathrm{~m}$} & LAGA & $7.567 \mathrm{E}+00$ & $7.552 \mathrm{E}+00$ & $1.42 \mathrm{E}-02$ & 220 \\
\hline & EACO & $7.549 \mathrm{E}+00$ & $7.533 \mathrm{E}+00$ & $9.42 \mathrm{E}-03$ & 102 \\
\hline & CGSA & $7.543 \mathrm{E}+00$ & $7.530 \mathrm{E}+00$ & $8.62 \mathrm{E}-03$ & 95 \\
\hline & FA & $7.528 \mathrm{E}+00$ & $7.510 \mathrm{E}+00$ & $9.55 \mathrm{E}-03$ & 78 \\
\hline & IBCO & $7.537 \mathrm{E}+00$ & $7.523 \mathrm{E}+00$ & 8.39E-03 & 84 \\
\hline & IBR-SFLA & $7.492 \mathrm{E}+00$ & $7.482 \mathrm{E}+00$ & $6.04 \mathrm{E}-03$ & 66 \\
\hline
\end{tabular}

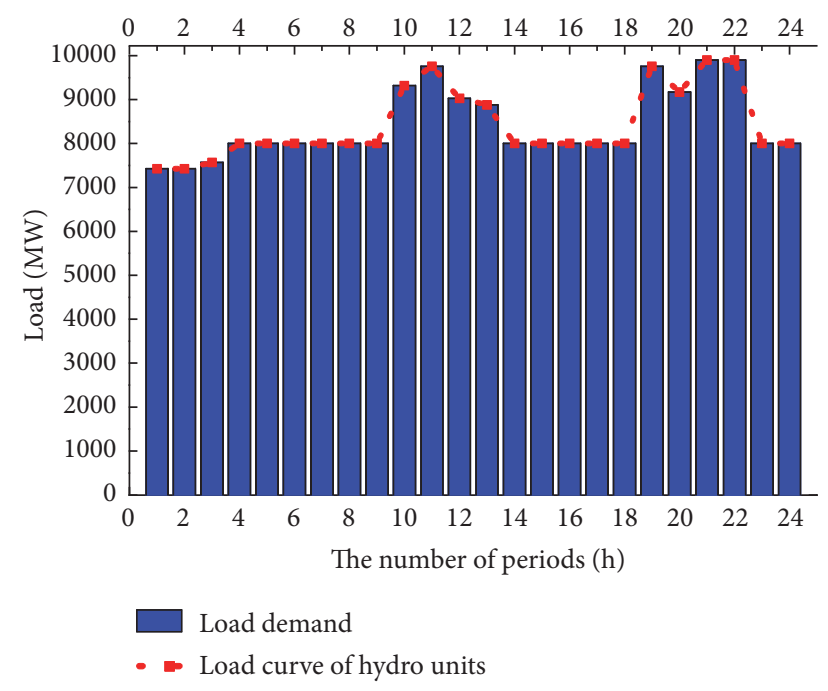

FIGURE 11: The daily load demand and real power output curve of hydropower station in the Three Gorges.

Table 5 gives details of total water consumption at each period corresponding to $75 \mathrm{~m}, 88 \mathrm{~m}$, and $107 \mathrm{~m}$ water head in which the water consumed by power generation and startup and shutdown of units is included.

To verify whether the power balance constraint (1) is satisfied, the comparison between daily load demand and real power output is presented in Figure 11 which reflects that real power output satisfies the load demand at each interval.
In order to profoundly demonstrate the superiority of IBR-SFLA in solving STHGS problem, a comparison experiment between IBR-SFLA and limited adaptive genetic algorithm (LAGA), extended ant colony optimization (EACO), chaotic gravitational search algorithm (CGSA), firefly algorithm (FA), and improved bee colony optimization (IBCO) is carried out under the same independent simulation times, iteration number, and population scale. The final optimal simulation results obtained by IBR-SFLA and other compared algorithms are listed in Figures 12 and 13. Figure 12 presents iterative process of total water consumption for each algorithm. From Figure 12, it can be observed the LAGA is less effective than other algorithms. The uncertainty of control parameters in LAGA dramatically affects the quality of solutions while the method IBR-SFLA proposed has relative less parameters we need to control. Results obtained by IBRSFLA are obviously more superior than others and water consumption descends drastically at the initial intervals and converges to stable optimal level at the later phase. The IBRSFLA outperforms the other algorithms through inspection of the convergence precision, which indicates the efficiency and adaption of IBR-SFLA in dealing with STHGS problem.

More details will be revealed if the results obtained by each algorithm in 20 independent simulations are expressed with form of box plot (Figure 13). The box plots present distribution of the simulation results from which IBR-SFLA exhibits significantly lower and narrower box plot with short whiskers than that in other rivals under $75 \mathrm{~m}$ and $88 \mathrm{~m}$ water heads. It can be stated that solutions obtained by IBR-SFLA in 20 simulations are higher quality and closer to the optimum than compared algorithms. In terms of 107 water heads, the 


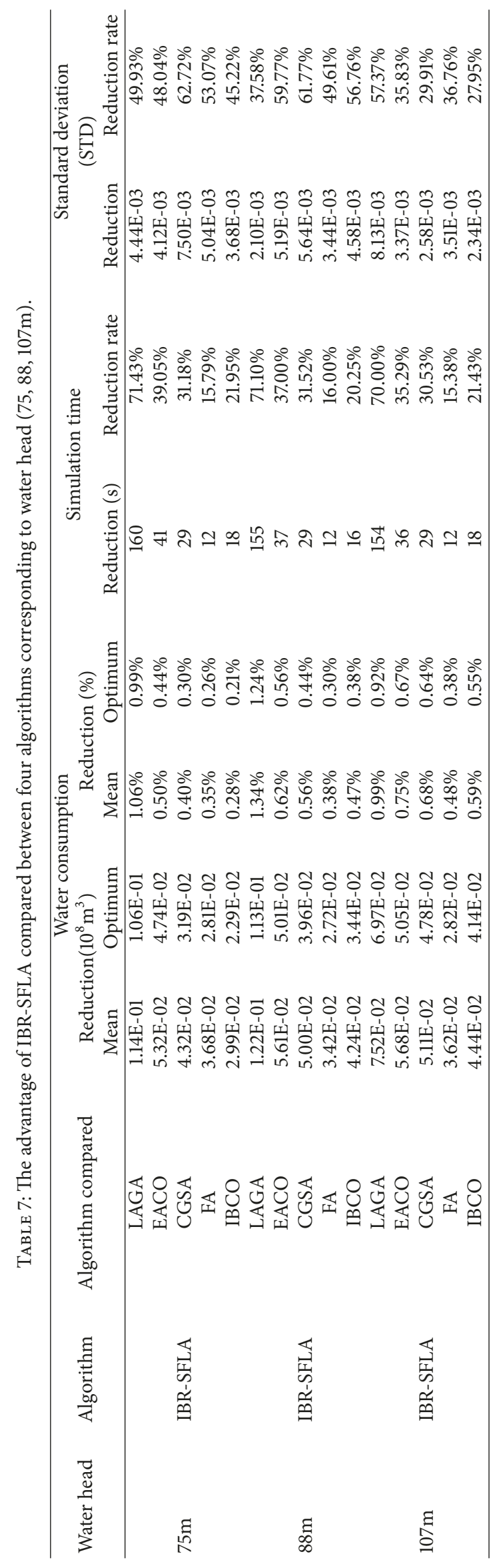



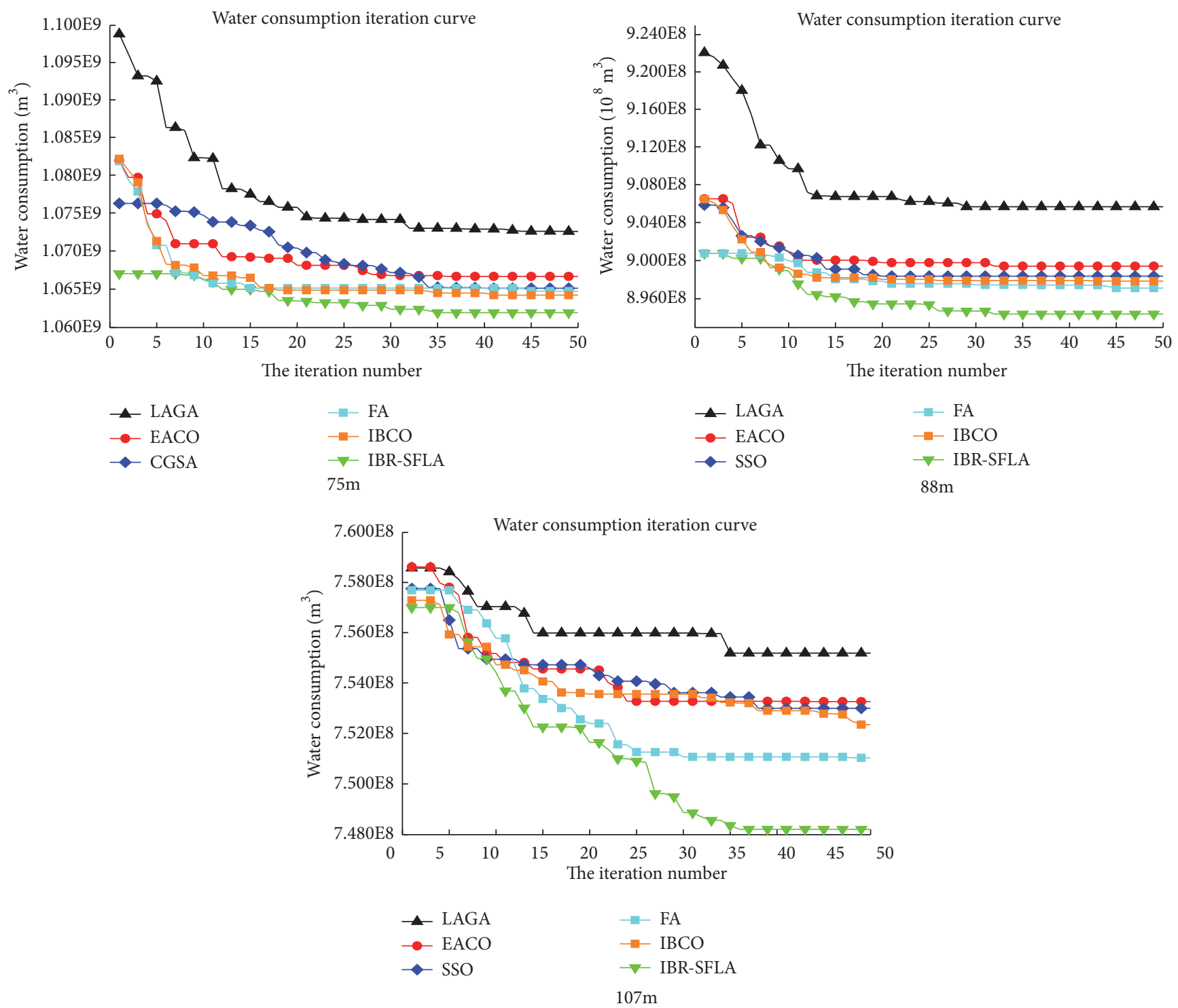

FIGURE 12: The iterative process of water consumption obtained by IBR-SFLA and compared algorithms corresponding to $75 \mathrm{~m}$, $88 \mathrm{~m}$, and $107 \mathrm{~m}$ water head.

boxes obtained using IBR-SFLA are lower than others but are not well at the coverage area of box plot. However, the whisker of box plot for IBR-SFLA is short which indicates that IBRSFLA outperforms other algorithms in convergence precision and reliability. To sum up, the higher quality results obtained by IBR-SFLA in 20 simulations demonstrate more effective and stable performance in comparison with other methods.

The results analysis simulated by each algorithm is listed in Table 6, in which the water consumption, standard deviation, and mean simulation time are shown simultaneously. The advantages of the IBR-SFLA compared with rivals are presented in Tables 6 and 7 and Figure 14. In terms of convergence precision, it is clear that IBR-SFLA can seek the less water consumption through more reasonable unit commitment and load dispatch. The results comparison demonstrates that the improved strategies introduced to
IBR-SFLA clearly improve the search capability and overall efficiency to converge to better quality solution.

Moreover, the simulation time cost by IBR-SFLA is observably less than other compared methods that reflect higher efficiency in dealing with STHGS for large scale hydropower station. The relative higher search speed has close relationship with optimizing mechanism of IBR-SFLA. Only the worst frog in frog subpopulations is updated during each iteration; therefore, only one calculation is needed to finish the iteration in the best case. Even if in the worst case, three calculations are required during each iteration.

In terms of algorithm stability, it can be concluded that IBR-SFLA has significant advantage over standard deviation (STD) compared with other algorithms. The smaller STD manifests that computational stability of IBR-SFLA is obviously improved and reflected by narrower box and shorter 

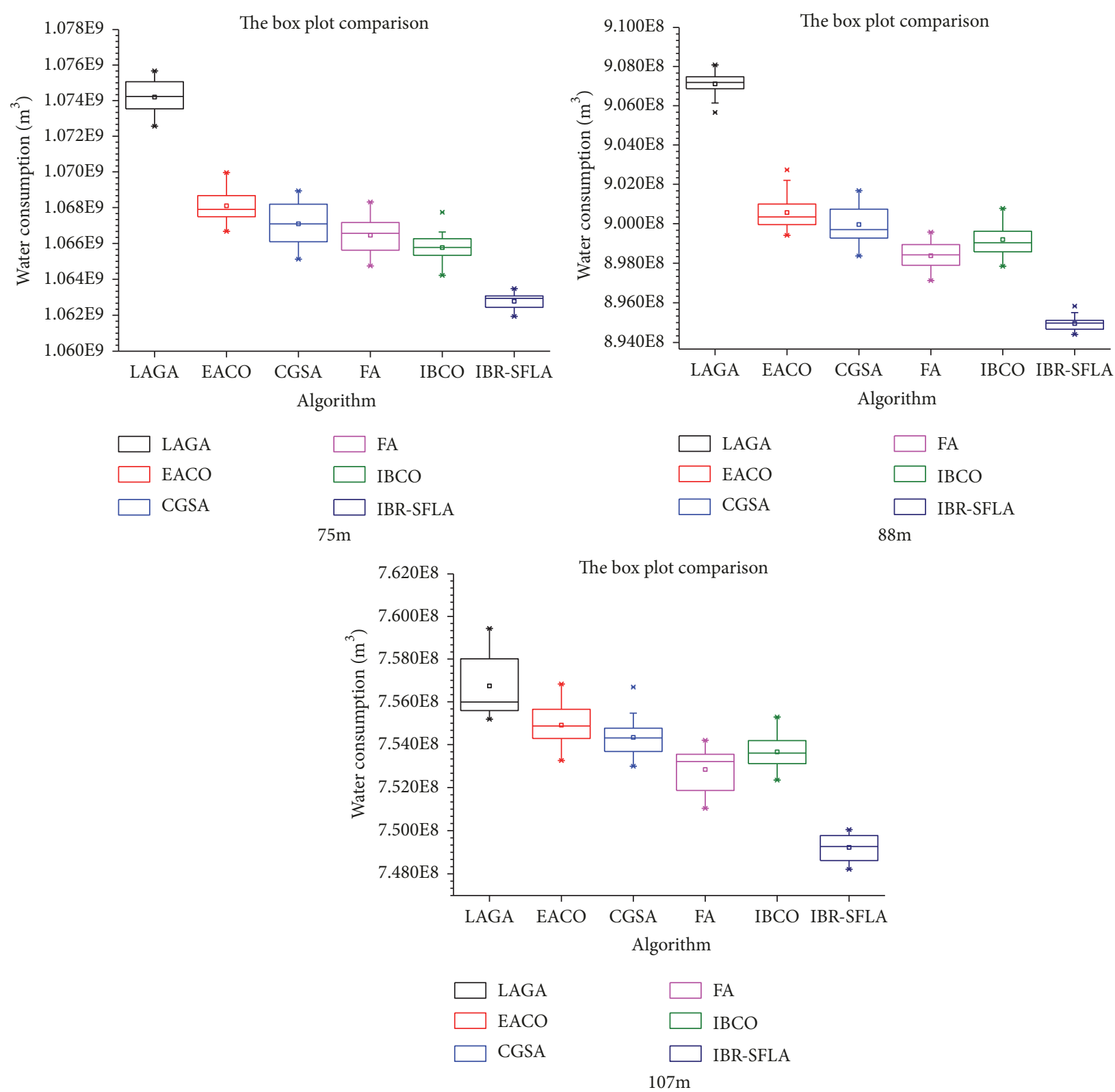

FIGURE 13: The box plots of water consumption in 20 simulations obtained by IBR-SFLA and compared algorithms.

whiskers in Figure 13. Consequently, the proposed IBR-SFLA is more likely to converge to final optimal solution in each simulation than other rivals.

In summary, IBR-SFLA shows higher solution quality, convergence speed, and reliability and stability in solving STHGS problem with complex hydraulic constraints. Therefore, the proposed IBR-SFLA is verified as a feasible and effective method to save water resources and further enhance waterpower utilization efficiency.

\section{Conclusions}

In this paper, an improved binary-real coded SFLA (IBRSFLA) is proposed for solving the STHGS problem for large scale hydropower station. To demonstrate the superiority of IBR-SFLA, numerical simulation experiment is conducted and results show IR-SFLA has exceptional performance on search capability and convergence speed. Then, IBR-SFLA is applied to the STHGS problem in Three Gorges hydropower station and heuristic repairing strategies are introduced to handle complex constraints such as minimum startup and shutdown, spinning reserve, and water balance. Simulation results indicate IBR-SFLA can get better water consumption solution with costing less computation time in comparison with compared methods. By further analysis, distribution of solutions obtained by IBR-SFLA is concentrated with less scatter, which verifies that stability and reliability of IBR-SFLA hold superiority compared to other methods. In conclusion, the proposed IBR-SFLA enhances overall 


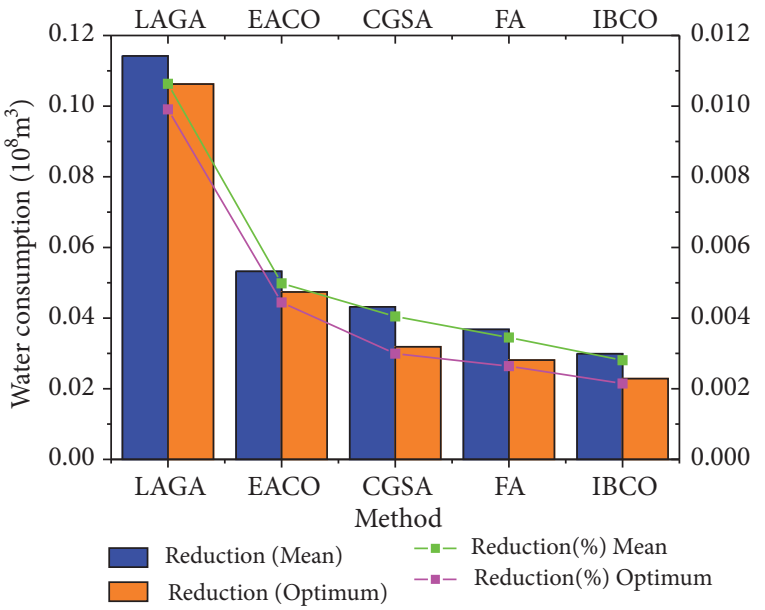

Water consumption

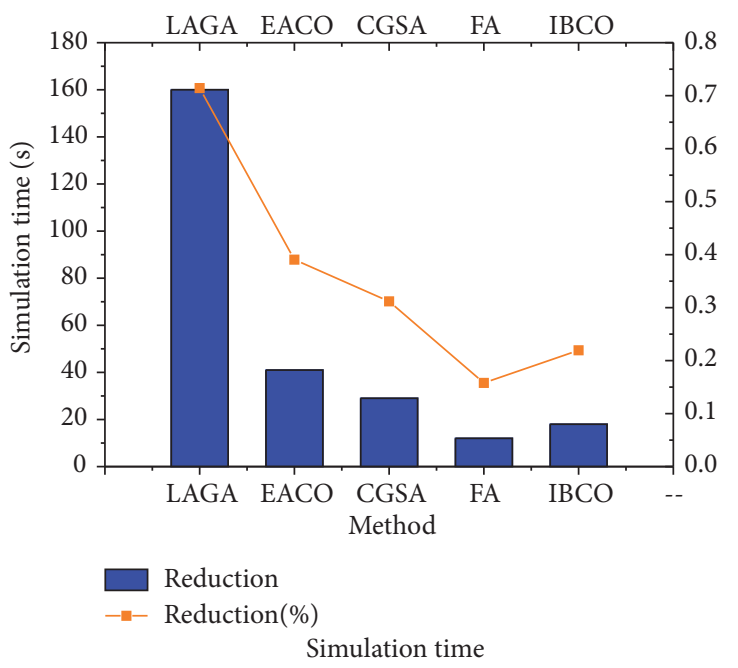

Simulation time

(a) $75 \mathrm{~m}$ water head

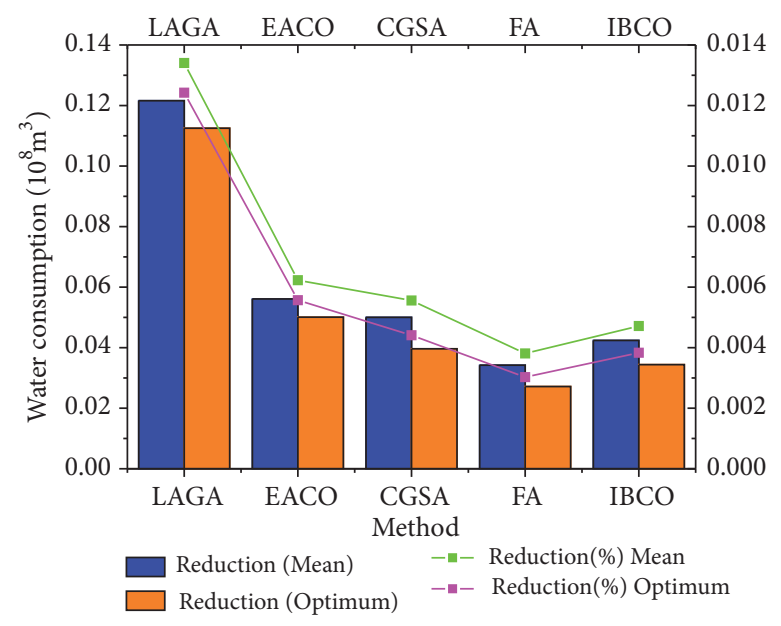

Water consumption

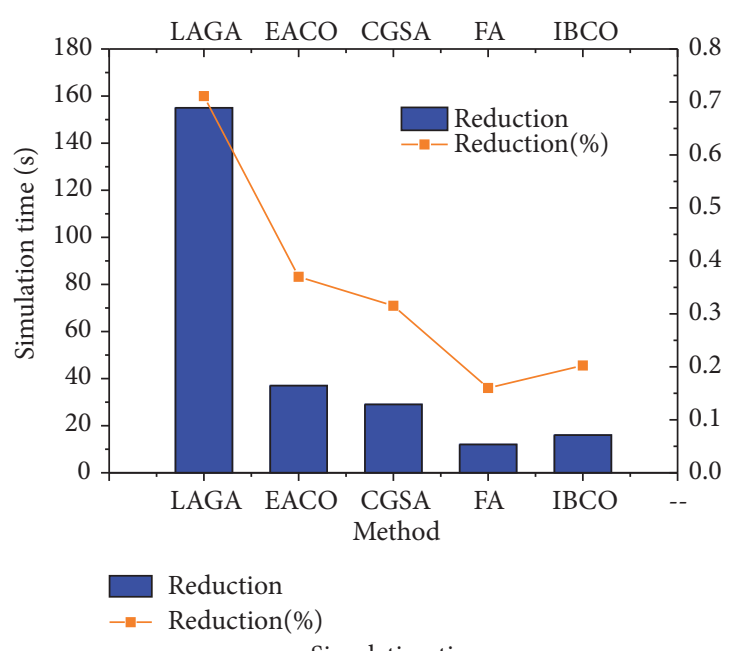

Simulation time

(b) $88 \mathrm{~m}$ water head

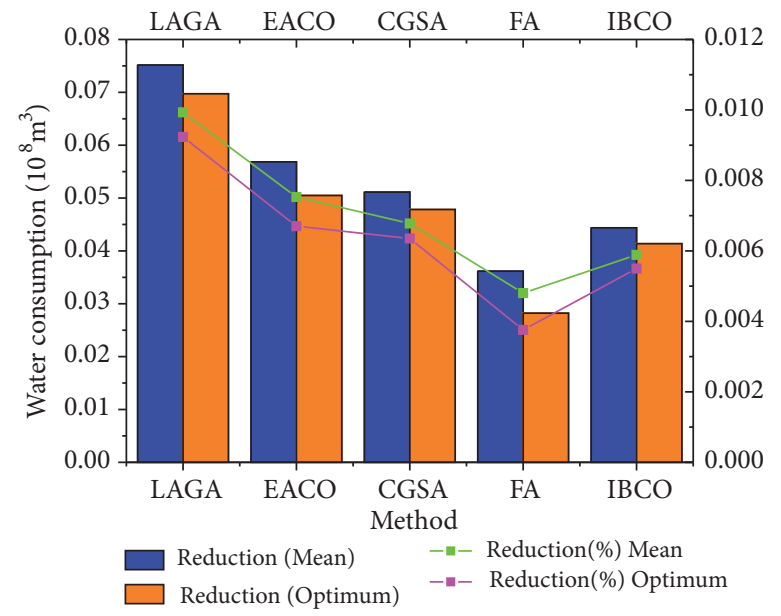

Water consumption

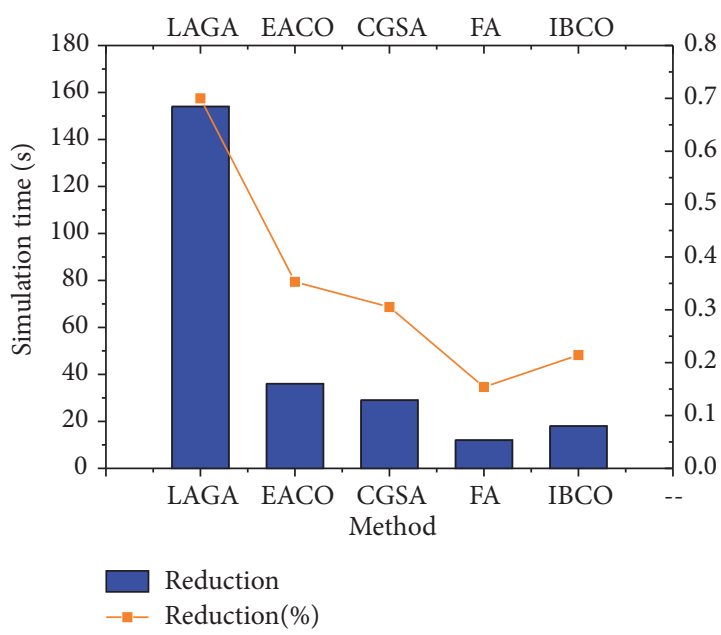

Simulation time

(c) $107 \mathrm{~m}$ water head

Figure 14: The contrasting result between four algorithms corresponding to three types of water head $(75,88$, and $107 \mathrm{~m})$. 
performance of traditional SFLA with more reasonable grouping strategy, effective search strategy based on cloud model, and chaotic theory. It is demonstrated that IBR-SFLA provides more effective and feasible way to solve STHGS problem with less water consumption and higher utilization rate of hydropower resources. In the future, related studies are focused on further improving convergence speed and enhancing comprehensive profitability for hydropower.

\section{Data Availability}

The data that support the findings of this study are available on request from the corresponding author, Professor Kan Yang.

\section{Disclosure}

This article does not contain any studies with human participants or animals performed by any of the authors and is in compliance with ethical standards.

\section{Conflicts of Interest}

The authors declare that there are no conflicts of interest.

\section{Acknowledgments}

The research was funded by the National Key Basic Research Program of China (973 Program) (2012CB417006) and the National Science Support Plan Project of China (2009BAC56B03).

\section{References}

[1] J. Zheng, K. Yang, and X. Lu, "Limited adaptive genetic algorithm for inner-plant economical operation of hydropower station," Hydrology Research, vol. 44, no. 4, pp. 583-599, 2013.

[2] W. Xiao, J. Z. Zhou, and Y. Q. Wang, "An improved BPSO based on dynamic inertia weight for automatic generation control," ICCNT, vol. 2, no. 3, pp. 510-514, 2011.

[3] O. Nilsson and D. Sjelvgren, "Hydro unit start-up costs and their impact on the short term scheduling strategies of swedish power producers," IEEE Transactions on Power Systems, vol. 12, no. 1, pp. 38-44, 1997.

[4] A. I. Cohen and M. Yoshimura, "A branch-and-bound algorithm for unit commitment," IEEE Transactions on Power Apparatus and Systems, vol. 102, no. 2, pp. 444-451, 1983.

[5] R. A. Jabr, A. Coonick, and B. Cory, "A homogeneous linear programming algorithm for the security constrained economic dispatch problem," IEEE Transactions on Power Systems, vol. 15, no. 3, pp. 930-936, 2000.

[6] J. P. S. Catalão, H. M. I. Pousinho, and V. M. F. Mendes, "Hydro energy systems management in Portugal: Profit-based evaluation of a mixed-integer nonlinear approach," Energy, vol. 36, no. 1, pp. 500-507, 2011.

[7] P. Oliveira, J. Blair-Fish, S. McKeeS, and C. Coles, "Parallel lagrangian relaxation in power scheduling," Computing Systems in Engineering, vol. 3, no. 5, pp. 609-612, 1992.
[8] W. Ongsakul and N. Petcharaks, "Unit commitment by enhanced adaptive Lagrangian relaxation," IEEE Transactions on Power Systems, vol. 19, no. 1, pp. 620-628, 2004.

[9] C.-P. Cheng and C.-W. Liu, "Unit commitment by Lagrangian Relaxation and Genetic Algorithms," IEEE Transactions on Power Systems, vol. 15, no. 2, pp. 707-714, 2000.

[10] T. Sum-Im, "Lagrangian relaxation combined with differential evolution algorithm for unit commitment problem," in Proceedings of the 19th IEEE International Conference on Emerging Technologies and Factory Automation, ETFA 2014, Spain, September 2014.

[11] G. P. Granelli and M. Montagna, "Security-constrained economic dispatch using dual quadratic programming," Electric Power Systems Research, vol. 56, no. 1, pp. 71-80, 2000.

[12] R. B. Allen and S. G. Bridgeman, "Dynamic programming in hydropower scheduling," Journal of Water Resources Planning and Management, vol. 112, no. 3, pp. 339-353, 1986.

[13] T. K. Siu, G. A. Nash, and Z. K. Shawwash, "A practical hydro, dynamic unit commitment and loading model," IEEE Transactions on Power Systems, vol. 16, no. 2, pp. 301-306, 2001.

[14] J. Yi, J. W. Labadie, and S. Stitt, "Dynamic optimal unit commitment and loading in hydropower systems," Journal of Water Resources Planning and Management, vol. 129, no. 5, pp. 388-398, 2003.

[15] C. T. Cheng, S. L. Liao, Z. T. Tang, and M. Y. Zhao, "Comparison of particle swarm optimization and dynamic programming for large scale hydro unit load dispatch," Energy Conversion and Management, vol. 50, no. 12, pp. 3007-3014, 2009.

[16] H. Wei and H. Qin, "Policy iteration-approximate dynamic programming for large scale unit commitment problems," Proceedings of the CSEE, vol. 34, no. 25, pp. 4420-4429, 2014.

[17] C. C. Asir Rajan, "Hydro-thermal unit commitment problem using simulated annealing embedded evolutionary programming approach," International Journal of Electrical Power \& Energy Systems, vol. 33, no. 4, pp. 939-946, 2011.

[18] Y. Lu, J. Zhou, H. Qin, Y. Wang, and Y. Zhang, "An adaptive chaotic differential evolution for the short-term hydrothermal generation scheduling problem," Energy Conversion and Management, vol. 51, no. 7, pp. 1481-1490, 2010, http://dx.doi.org/10.1016/j.enconman.2010.02.006.

[19] L. Mo, P. Lu, C. Wang, and J. Zhou, "Short-term hydro generation scheduling of Three Gorges-Gezhouba cascaded hydropower plants using hybrid MACS-ADE approach," Energy Conversion and Management, vol. 76, pp. 260-273, 2013, http://dx.doi.org/10.1016/j.enconman.2013.07.047.

[20] M. A. Abido, "A niched Pareto genetic algorithm for multiobjective environmental/economic dispatch," International Journal of Electrical Power \& Energy Systems, vol. 25, no. 2, pp. 97-105, 2003.

[21] V. Senthil Kumar and M. R. Mohan, "Solution to security constrained unit commitment problem using genetic algorithm," International Journal of Electrical Power \& Energy Systems, vol. 32, no. 2, pp. 117-125, 2010.

[22] N. Bakhta, B. Hamid, and A. Ahmed, "Solving the economic dispatch problem by using tabu search algorithm," Energy Procedia, vol. 36, pp. 694-701, 2013.

[23] H. Sasaki, M. Watanabe, J. Kubokawa, N. Yorino, and R. Yokoyama, "A Solution Method of Unit Commitment by Artificial Neural Networks," IEEE Transactions on Power Systems, vol. 7, no. 3, pp. 974-981, 1992. 
[24] X. Yuan, H. Nie, A. Su, L. Wang, and Y. Yuan, "An improved binary particle swarm optimization for unit commitment problem," Expert Systems with Applications, vol. 36, no. 4, pp. 8049$8055,2009$.

[25] R. Kumar, D. Sharma, and A. Sadu, "A hybrid multi-agent based particle swarm optimization algorithm for economic power dispatch," International Journal of Electrical Power \& Energy Systems, vol. 33, no. 1, pp. 115-123, 2011.

[26] H. L. Chen and R. P. Guo, "Unit commitment based on improved discrete particle swarm optimization," Power System Technology, vol. 35, no. 12, pp. 94-99, 2011.

[27] J. Cai, X. Ma, L. Li, and P. Haipeng, "Chaotic particle swarm optimization for economic dispatch considering the generator constraints," Energy Conversion and Management, vol. 48, no. 2, pp. 645-653, 2007.

[28] M. W. Wang and L. Yang, "Unit commitment based on improved immune algorithm," Power System Technology, vol. 34, no. 8, pp. 112-117, 2010.

[29] K. Vaisakh and L. R. Srinivas, "Evolving ant colony optimization based unit commitment," Applied Soft Computing, vol. 2, no. 11, pp. 2863-2870, 2011, http://dx.doi.org/10.1016/j.asoc.2010.11.019.

[30] L. Chen, K. Yang, and H. Li, "Overall temporal and spatial economic operation model and it's algorithm for large-scale hydropower station," Journal of Huazhong University of Science and Technology (Natrural Science Edition), vol. 43, no. 9, pp. 117122, 2015.

[31] D. M. Shen, Research on Unit Commitment Problems in Power System Based on Improved Gravitational Search Algorithm, Donghua University, 2016.

[32] D. M. Shen, T. Jiang, W. Chen, Q. Shi, and S. C. Gao, "Improved chaotic gravitational search algorithms for global optimization," in Proceedings of the IEEE Congress on Evolutionary Computation(CTC), pp. 1220-1226, Sendai, Japan, 2015.

[33] X. Yuan, B. Ji, S. Zhang, H. Tian, and Y. Hou, "A new approach for unit commitment problem via binary gravitational search algorithm," Applied Soft Computing, vol. 22, pp. 249-260, 2014.

[34] C. Li, J. Zhou, P. Lu, and C. Wang, "Short-term economic environmental hydrothermal scheduling using improved multiobjective gravitational search algorithm," Energy Conversion and Management, vol. 89, pp. 127-136, 2015.

[35] K. S. Reddy, L. K. Panwar, R. Kumar, and B. K. Panigrahi, "Binary fireworks algorithm for profit based unit commitment (PBUC) problem," International Journal of Electrical Power \& Energy Systems, vol. 83, pp. 270-282, 2016.

[36] F. Hu, D. H. Zhang, and X. C. Yang, "Optimal load distribution and comitment of automatic generation control units in hydropower station based on ant colony algorithm," Water Resources and Power, vol. 30, no. 12, pp. 123-126, 2012.

[37] F. Wu, Economic Dispatch for Wind-Thermal Hybrid Power Systems Based on Particle Swarm Optimization, North China Electric Power University, 2015.

[38] M. Eusuff, K. Lansey, and F. Pasha, "Shuffled frog-leaping algorithm: a memetic meta-heuristic for discrete optimization," Engineering Optimization, vol. 38, no. 2, pp. 129-154, 2006.

[39] P. Sun, Z.-Q. Jiang, T.-T. Wang, and Y.-K. Zhang, "Research and application of parallel normal cloud mutation shuffled frog leaping algorithm in cascade reservoirs optimal operation," Water Resources Management, vol. 30, no. 3, pp. 1019-1035, 2016.

[40] J. Cao, Research on the Unit Commitment Problems in Coal-Fired Power Plants Based on Shuffled Frog Leaping Algorithm, Ningxia University, 2014.
[41] H. Li and W. Yin, "An improved SFLA algorithm for economic operation of hydroelectric plant," Water Conservancy Science and Technology and Economy, vol. 20, no. 2, pp. 127-130, 2014.

[42] P. Lu, J. Zhou, C. Wang, Q. Qiao, and L. Mo, "Short-term hydro generation scheduling of Xiluodu and Xiangjiaba cascade hydropower stations using improved binaryreal coded bee colony optimization algorithm," Energy Conversion and Management, vol. 91, pp. 19-31, 2015, http://dx.doi.org/10.1016/j.enconman.2014.11.036.

[43] F. Q. Zhao and Z. G. Chen, "Shuffled frog-leaping algorithm based on the theory of adaptive mutation factors," Journal of Gansu Sciences, vol. 28, no. 1, pp. 6-11, 2016.

[44] C. R. Zou, X. D. Zhang, and L. Zhao, "Review of shuffled frog leaping algorithm," Electronic Engineer, vol. 38, no. 5, pp. 1-5, 2012.

[45] J. Luo, X. Li, M.-R. Chen, and H. Liu, "A novel hybrid shuffled frog leaping algorithm for vehicle routing problem with time windows," Information Sciences, vol. 316, pp. 266-292, 2015, http://dx.doi.org/10.1016/j.ins.2015.04.001.

[46] D. Y. Li, H. J. Meng, and X. M. Shi, "Membership clouds and membership cloud generators," Journal of Computer Research and Development, vol. 32, no. 6, pp. 15-20, 1995.

[47] C. Dai, Y. Zhu, and W. Chen, "Cloud model based genetic algorithm and its applications," Acta Electronica Sinica, vol. 35, no. 7, pp. 1419-1424, 2007.

[48] C. L. Zhang, C. R. Yu, S. H. Jiang, Q. J. Liu, W. J. Wu, and Y. M. Li, "Particle swarm optimization algorithm based on chaos cloud model," Journal of Computer Application, vol. 32, no. 7, pp. 1951-1954, 2012.

[49] G. Zhang, R. He, and Y. Liu, "An evolutionary algorithm based on cloud model," Chinese Journal of Computers, vol. 31, no. 7, pp. 1082-1091, 2008 (Chinese).

[50] Y. Ma, W. J. Tian, and Y. Y. Fan, "Quantum adaptive immune clone algorithm based on cloud model," Chinese Journal of Computational Physics, vol. 30, no. 4, pp. 627-632, 2013.

[51] H. Ding and D. Wang, "The evaluation method of water eutrophication based on cloud model," Huanjing Kexue Xuebao/Acta Scientiae Circumstantiae, vol. 33, no. 1, pp. 251-257, 2013.

[52] Q. Zhang and P. C. Li, "Adaptive grouping chaotic cloud model shuffled frog leaping algorithm fror continuous space optimization problems," Control and Decision, vol. 30, no. 5, pp. 923-928, 2015

[53] Q. Zou, X. M. Wang, A. Q. Li, X. C. He, and B. Luo, "Optimal operation of flood control for cascade reservoirs based on parallel chaotic quantum particle swarm optimization," Journal of Hydraulic Engineering, vol. 47, no. 8, pp. 967-976, 2016.

[54] C.-T. Cheng, W.-C. Wang, D.-M. Xu, and K. W. Chau, "Optimizing hydropower reservoir operation using hybrid genetic algorithm and chaos," Water Resources Management, vol. 22, no. 7, pp. 895-909, 2008.

[55] Z. J. Teng and X. Y. Zhang, "The layout optimization of WSN based on inertia weight shuffled frog leaping algorithm," Journal of Northeast Dianli University, vol. 35, no. 6, pp. 66-69, 2015. 


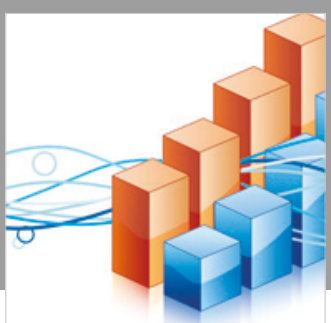

Advances in

Operations Research

\section{-n-m}
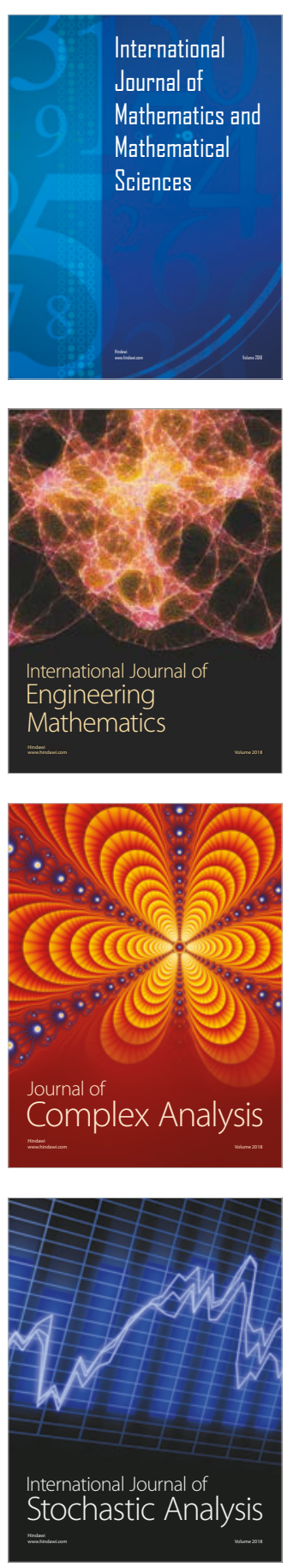
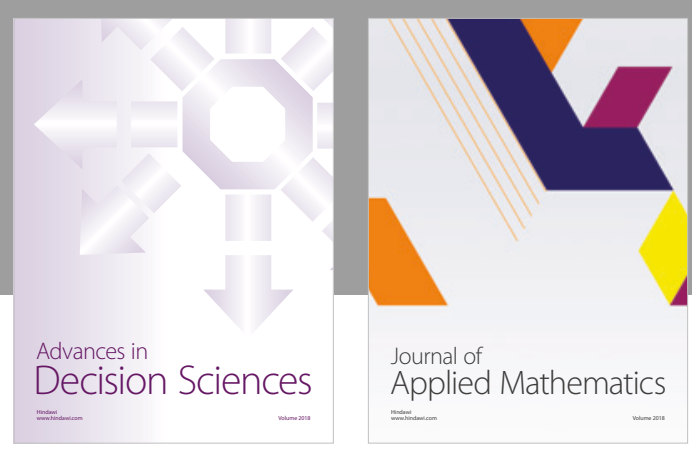

Journal of

Applied Mathematics
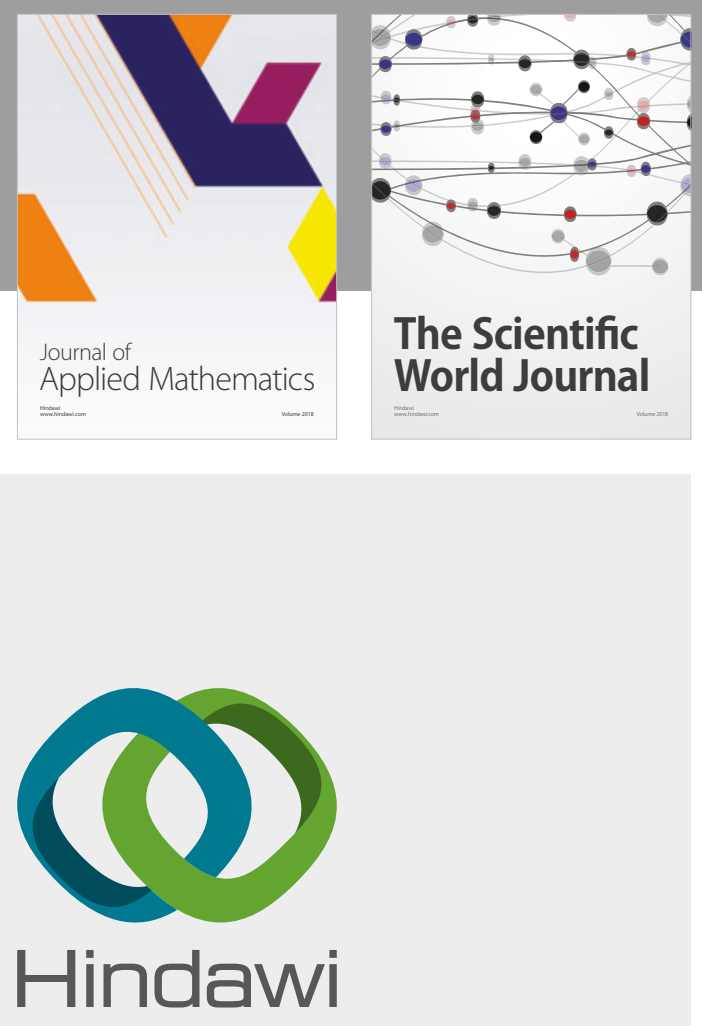

Submit your manuscripts at

www.hindawi.com

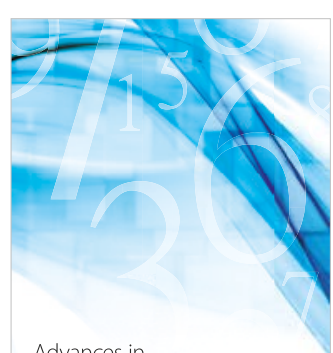

Advances in
Numerical Analysis
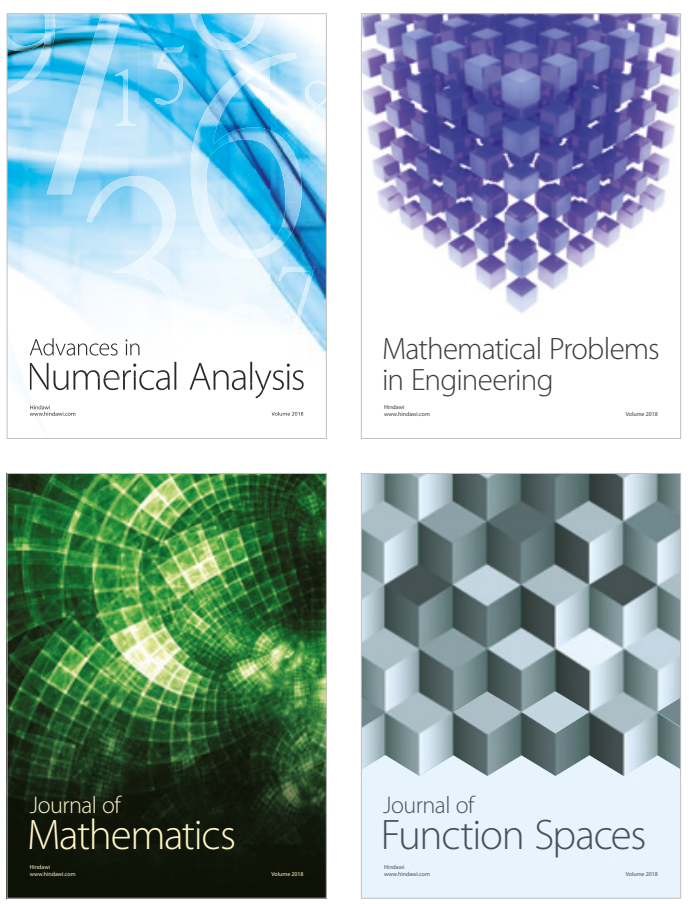

Mathematical Problems in Engineering

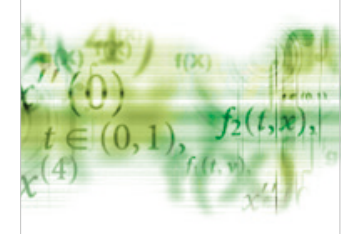

International Journal of

Differential Equations

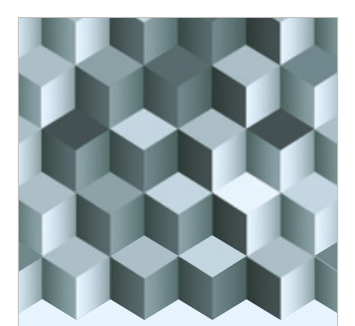

Journal of

Function Spaces

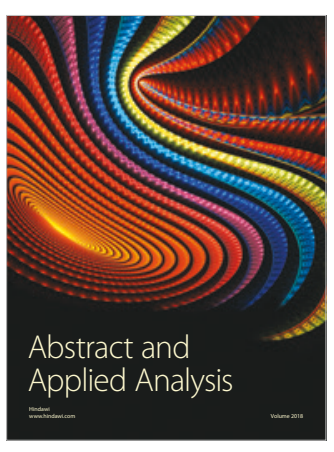

The Scientific

World Journal

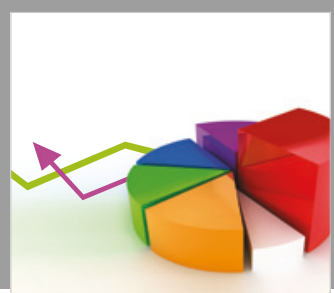

Journal of

Probability and Statistics
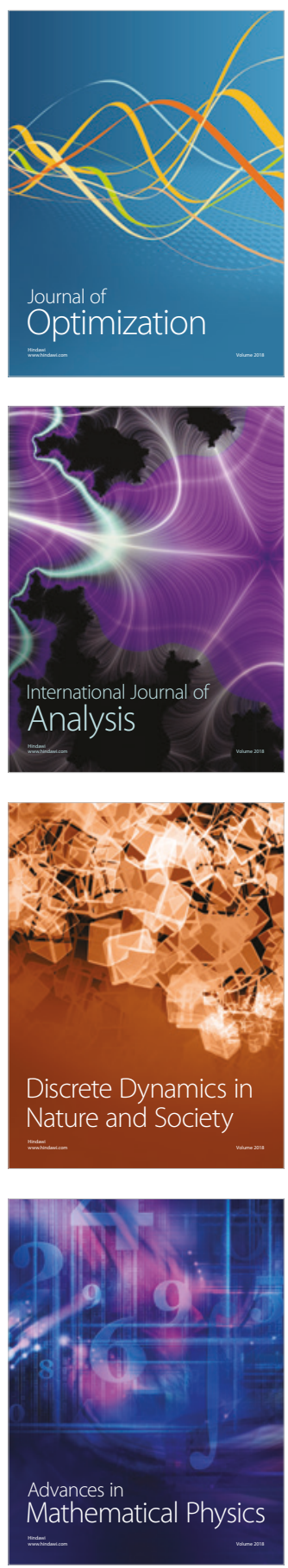